\title{
APPLICATION OF REGRESSION, VARIANCE, AND DENSITY BASED GLOBAL SENSITIVITY METHODS TO COUPLED VERA-CS AND BISON SIMULATIONS
}

Hongbin Zhang, Charlie Folsom, Heng Ban, Ronaldo Szilard, Cole Blakely

June 2018

The INL is a U.S. Department of Energy National Laboratory operated by Battelle Energy Alliance

Idaho National Laboratory 


\section{APPLICATION OF REGRESSION, VARIANCE, AND DENSITY BASED GLOBAL SENSITIVITY METHODS TO COUPLED VERA-CS AND BISON SIMULATIONS}

Hongbin Zhang, Charlie Folsom, Heng Ban, Ronaldo Szilard, Cole Blakely

June 2018

Idaho National Laboratory Idaho Falls, Idaho 83415

http://www.inl.gov

Prepared for the

U.S. Department of Energy

Under DOE Idaho Operations Office

Contract DE-AC07-05ID14517 


\title{
APPLICATION OF REGRESSION, VARIANCE, AND DENSITY BASED GLOBAL SENSITIVITY METHODS TO INTEGRATED VERA-CS AND BISON SIMULATIONS
}

\author{
Cole Blakely', Hongbin Zhang,", Charlie Folsom², Heng Ban³, Ronaldo Szilard ${ }^{2}$ \\ ${ }^{1}$ Department of Mechanical and Aerospace Engineering, \\ Utah State University, Old Main Hill, Logan, UT 84322 \\ Cole.Blakely@inl.gov \\ ${ }^{2}$ Idaho National Laboratory, 2525 Fremont Ave., Idaho Falls, ID 83415-3870 \\ Hongbin.Zhang@inl.gov, Charlie.Folsom@inl.gov, Ronaldo.Szilard@inl.gov \\ ${ }^{3}$ Department of Mechanical Engineering \& Materials Science \\ University of Pittsburgh, 3700 O'Hara Street, Pittsburgh, PA 15261
}

Heng.Ban@pitt.edu

\begin{abstract}
Uncertainty quantification (UQ) and sensitivity analyses (SA) are performed for coupled simulations between VERA-CS, a coupled pin resolved neutron transport and subchannel thermal hydraulics code, and the fuel performance code BISON. The interface between VERA-CS and BISON is performed in a multiphysics environment known as the LOCA Toolkit for U.S. light water reactors (LOTUS) currently under development at Idaho National Laboratory (INL). A focus is placed on using a variety of SA measures, including two regression based (Pearson and Spearman), one variance based (Sobol indices), and three moment independent measures (Delta moment independent measures with $\mathrm{L}_{1}, \mathrm{~L}_{2}$, and $\mathrm{L}_{\infty}$ norms). The problem under inspection is a single assembly depletion case for three fuel cycles. The figures of merit are the minimum departure from nucleate boiling ratio (MDNBR), maximum fuel centerline temperature (MFCT), and gap conductance at peak power (GCPP). SA results show MDNBR to be linear with consistent rankings throughout the fuel cycles. MFCT is linear, but with a change in rankings at the switch from open gap to closed gap models. GCPP is nonlinear at intermediate states that coincide with the onset of contact between fuel and cladding. These nonlinear states allow for the showcasing of higher order SA measures over first order methods.
\end{abstract}

KEYWORDS

Multiphysics Environment, Uncertainty Quantification, Sensitivity Analysis, VERA-CS, BISON

$\begin{array}{ll}\text { ABREVIATIONS } \\ \text { BEPU } & \text { Best Estimate Plus Uncertainty } \\ \text { CASL } & \text { Consortium for Advanced Simulation of Light Water Reactors } \\ \text { CIPS } & \text { Crud Induced Power Shift } \\ \text { CD-A } & \text { Core Design Automation } \\ \text { CD-O } & \text { Core Design Optimization } \\ \text { COBRA-TF } & \text { Coolant-Boiling in Rod Arrays-Two Fluids } \\ \text { FOM } & \text { Figure Of Merit } \\ \text { GCPP } & \text { Gap Conductance at Peak Power }\end{array}$

${ }^{*}$ Corresponding author. 


$\begin{array}{ll}\text { HPC } & \text { High Performance Computers } \\ \text { INL } & \text { Idaho National Laboratory } \\ \text { LOCA } & \text { Loss Of Coolant Accident } \\ \text { LOTUS } & \text { LOCA Toolkit for U.S. Light Water Reactors } \\ \text { MCNP } & \text { Monte Carlo N-Particle } \\ \text { MFCT } & \text { Maximum Fuel Centerline Temperature } \\ \text { MDNBR } & \text { Minimum Departure from Nucleate Boiling Ratio } \\ \text { MPACT } & \text { Michigan Parallel Characteristics Transport Code } \\ \text { MOOSE } & \text { Multiphysics Object Oriented Simulation Environment } \\ \text { NEAMS } & \text { Nuclear Energy Advanced Modeling and Simulation } \\ \text { NEM } & \text { Nodal Expansion Method } \\ \text { ORNL } & \text { Oak Ridge National Laboratory } \\ \text { PDF } & \text { Probability Distribution Function } \\ \text { PWR } & \text { Pressurized Water Reactor } \\ \text { RA } & \text { Risk Assessment } \\ \text { RIA } & \text { Reactivity Insertion Accident } \\ \text { SA } & \text { Sensitivity Analysis } \\ \text { UQ } & \text { Uncertainty Quantification } \\ \text { VERA-CS } & \text { Virtual Environment for Reactor Analysis - Core Simulator }\end{array}$

\section{INTRODUCTION}

The current fleet of the U.S. nuclear power plants is expected to undergo a variety of technological developments including the use of accident tolerant fuel and higher burnup fuel to improve its economic performance. In order to help facilitate this shift, improved predictive methods are currently under development, in particular multiphysics environments.

The word multiphysics indicates a set of calculations involving multiple physical models. It is worth noting that it is rather arbitrary when a simulation enters the realm of multiphysics, as most engineering codes model multiple physical phenomena. For instance, legacy codes such as FRAPCON solve mechanical and thermal equilibrium equations as well as irradiative effects, oxidation, thermal hydraulics, and fission gas release. However, code documentation for FRAPCON [1] never refers to itself as a multiphysics code. This is partly due to the term being less common during the development of FRAPCON, but also due to the fact that all the phenomena receiving particular focus reside within the fuel performance discipline. The usage of multiphysics within this work implies the combining of interdisciplinary calculations which have commonly been treated separately in the past.

The definition of an environment in the computational context is amorphous as well noted by Sloan et al. [2]. In this work, the word environment indicates a computational space in which users may utilize a variety of tools to generate, exchange, and process large amounts of data. Within this work, an environment is created that treats codes as modules which can readily and reliably be called upon in order to perform large Monte Carlo samplings. This environment allows for the collaboration of multiple large scale projects for a more thorough modeling of designs to be employed in the nuclear industry.

Multiphysics studies have been implemented and studied extensively. In nuclear applications, work on multiphysics tends to focus on combining fuel performance, thermal hydraulics, neutronics, and/or isotopic depletion codes. Two multiphysics environments utilized by this work are the Virtual Environment for Reactor Analysis - Core Simulator (VERA- CS) [3] developed by the Consortium for Advanced Simulation of Light Water Reactors (CASL) and the Multiphysics Object-Oriented Simulation Environment (MOOSE) developed at Idaho National Labs (INL). The version of VERA-CS used in this work performs tightly coupled calculations between the neutronics code MPACT, the thermal hydraulic 
code CTF, and the isotopic depletion code ORIGEN (see section 2.1). MOOSE uses a modularized approach to allow for increased flexibility in code development. MOOSE employs fully-coupled, fullyimplicit numerical methods to solve nonlinear equations within an adaptable, finite element framework [4] (see section 2.2). This work uses the fuel performance code BISON, which is developed within MOOSE.

Notable instances of thermal hydraulics and neutronics coupling include the thermal hydraulics code Trace-PFI with a neutronics code based on the nodal expansion method (NEM) [5] and a coupling of Monte Carlo based neutronics with subchannel thermal hydraulics [6]. Examples of fuel performance and neutronics coupling include the fuel performance code TRANSURANUS and neutronics code DYN3D [7], and BISON with DeCART [8]

Couplings between fuel performance and thermal hydraulics include FRAPCON with TRACE-V5P3 [9] and BISON [10] with RELAP5-3D [11]. Furthermore a coupling between the transient fuel performance code FRAPTRAN, subchannel thermal hydraulics code COBRA-TF, and the neutronics code TORT-TD has been performed [12]. A notable instance of a coupling between isotopic depletion and neutronics is the MCWO script [13] developed at INL which couples the isotopic depletion code ORIGEN2 with the Monte Carlo N-Particle transport code MCNP.

The Serpent Monte Carlo code [14] developed at the VTT technical center is also noteworthy for its widespread use in multiphysics studies, which include couplings of thermal hydraulics, computational fluid dynamics, isotopic depletion, fuel performance, and neutron and photon transport. Considerable work is also underway in the U.S. Department of Energy's program known as Nuclear Energy Advanced Modeling Simulation (NEAMS) to develop a workbench [15] to handle the integration of neutronics, fuel performance, thermal hydraulics, and isotopic depletion codes. Finally the reactor and lattice physics code SCALE [16] developed by Oak Ridge National Laboratory (ORNL) has been coupled with thermal hydraulic, computational fluid dynamics, and fuel performance codes. It is worth noting that the specified problem (i.e. reactivity insertion accident (RIA), loss of coolant accident (LOCA), quasi-steady state depletion) often varies among the aforementioned works.

An essential aspect in the development of multiphysics environments is the incorporation of uncertainty quantification (UQ) and sensitivity analysis (SA) methodologies. UQ and SA studies of multiphysics environments ensure that the results are not treated as absolutes, but rather as estimates with inherent uncertainty. In essence, UQ and SA studies assure that decision makers are privy to the best estimate plus uncertainty (BEPU) methodology which has become the de-facto industry standard.

UQ and SA in reactor design and analysis had predominantly been used in the field of thermal hydraulics $[17,18]$, but recently have been extended to fuel performance $[19,20]$. UQ and SA have been performed on the fuel performance code BISON [21, 22] using DAKOTA software, as well as with the RAVEN software developed at INL [23]. UQ and SA have also been extended to VERA-CS [24, 25]. UQ has also been performed on VERA-CS via the Crud Induced Power Shift studies (CIPS) [26]. Notable UQ and SA have also been performed on SCALE [27]. UQ and SA on large multiphysics environments are still at their infancy and warrant further systematic studies.

In this work, a Monte Carlo based UQ and SA have been performed on an integration of CASL's VERACS code [3] and INL's advanced fuel performance code BISON [10]. The integration of VERA-CS and BISON and the subsequent UQ/SA studies are performed within the context of a multiphysics environment known as LOTUS which stands for LOCA Toolkit for the U.S. light water reactors.

For each instance in the Monte Carlo sampling, a VERA-CS output file was generated, from which the power histories and thermal hydraulic profiles for a pin of interest were inputted into BISON. The pin of 
interest in this case was defined as the pin with the highest linear heat rate at the end of the fuel life cycle. This selection criteria was chosen due to the fact that the fuel performance figures of merit (FOM) of this work always coincided with the highest power pin for all observed cases. While the maximum power pin changes location throughout the fuel cycle, a consistent pin location based solely on the maximum pin power at end of three fuel cycles was used so as to maintain consistency. The outputs from VERA-CS and BISON were then used to obtain the FOM, from which all UQ and SA were performed.

The problem of interest presented in this work is a three cycle, single assembly, fuel depletion case. This case was selected based on its similarities with typical operational behavior of an assembly in a LWR. While ideally a full core would be simulated, a single assembly is more feasible for a Monte Carlo sampling given current computational limits. The quasi-steady depletion case will also eventually serve as a means of supplying initial conditions (particularly gap conductance) to LOCA cases which are to be the primary focus of future LOTUS studies as the name suggests.

The FOM for this case are the minimum departure from nucleate boiling ratio (MDNBR), maximum fuel centerline temperature (MFCT), and the gap conductance at peak power (GCPP). The GCPP results from BISON are of particular interest as past work with FRAPCON [28], showed GCPP uncertainty to be highly nonlinear. This nonlinear behavior is likely attributable to abrupt shifts in certain empirical functions used within FRAPCON, as opposed to the more mechanistic models used within BISON (see section 2.2).

All SA studies must decide upon which SA measure to employ and report among an ever expanding list of possible candidates. Each SA measure has advantages and disadvantages typically in the realms of required sample size and the ability to detect nonlinearities. The LOTUS environment provides a post processing toolkit for the calculation of a variety of SA measures for any set of recorded inputs and corresponding outputs. This work employs two regression based SA measures (Pearson and Spearman), one variance based (Sobol indices), and three moment independent measures (Delta Moment Independent [29] with, $\mathrm{L}_{2}$ and $\mathrm{L}_{\infty}$ norm variations [30]).

Results show MDNBR to behave linearly and consistently among all SA measures and states throughout the fuel cycle. The most impactful inputs for MDNBR are the assembly power, inlet temperature, and outlet pressure. Lesser contributions also come from rated flow and clad outer diameter.

MFCT also behaves linearly with consistency among SA measures, but with significant shifts in SA measures from state to state. Initially, MFCT depends primarily on fuel thermal conductivity in addition to cladding inner radius and fuel radius due to their direct effect on gap conductance. Uncertainties of later states stem almost exclusively from fuel thermal conductivity, with lesser contributions from fuel density and assembly power due to their effect on burnup which in turn effects fuel thermal conductivity.

GCPP contains a variety of nonlinearities allowing for differences among SA measures which highlight their unique benefits. At the start of the fuel cycle, GCPP is relatively linear with a strong dependence on fuel radius and clad inner radius due to their direct role in gap width. Near the end of the first fuel cycle, many cases are a mixture of open and closed gap models, causing nonlinear relations. Impactful inputs at this state are the fuel and inner cladding radii, fuel and cladding roughness (as a result of the gap width used in closed gap modeling), and gap gas thermal conductivity. Later states show a return to linearity as all cases become closed gap, with no interaction from initial gap width, and larger dependencies on surface roughness and fission gas release diffusion coefficients.

The purpose of this work is to demonstrate the LOTUS environment and better understand the uncertainty of the aforementioned FOM. The LOTUS demonstration will serve as a starting point from which later work on code integrations can be performed, specifically for accident tolerant fuel and higher burnup fuel 
simulations. The FOM subject to UQ and SA were selected in the interests of eventually narrowing reactor safety margins (see section 4.1). In particular, insights gained from the study of MFCT and GCPP will be of value in later LOCA studies. Performing BEPU methodology with a Monte Carlo based UQ within the multiphysics framework of LOTUS creates a less conservative estimate as compared to the more commonly used Wilks' method. This improved estimate has the potential to allow higher operational power and/or greater flexibility when performing power maneuvers.

\section{LOTUS MULTIPHYSICS ENVIRONMENT}

The larger vision for LOTUS is to have a single multiphysics environment with the ability to perform Core Design-Automation (CD-A), Fuel Performance, Systems Analysis, Risk Assessment (RA), and Core Design-Optimization (CD-O). A conceptual layout for LOTUS is shown in Figure 1.

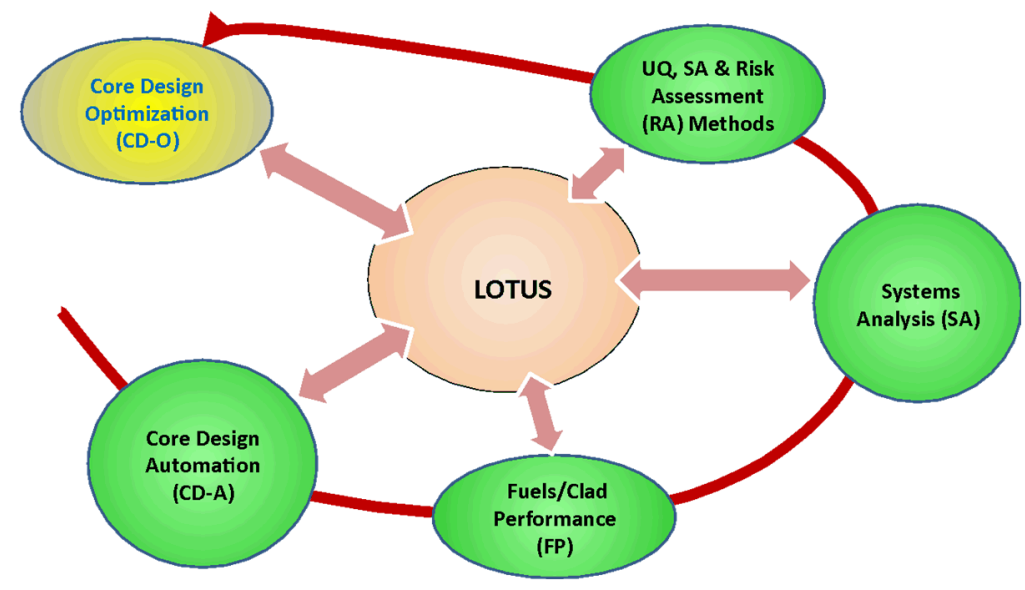

Figure 1. Schematic illustration of LOTUS

The focus of this work is a subset of the LOTUS framework development which involves the coupling of the core design automation code VERA-CS with the fuel performance code BISON. While the modeling of LOCA is the eventual goal of LOTUS, this is not possible without supplied initial conditions from steady state simulations. Thus, while future work will center on LOCA, present work focuses on the preliminary step of ascertaining data from quasi steady state depletion cases (see section 4).

\subsection{VERA-CS}

VERA-CS employs MPACT for neutron transport, COBRA-TF for subchannel thermal hydraulics, and ORIGEN for isotopic depletions [3]. Due to the coupling of these codes, VERA-CS is a multiphysics environment for reactor core simulations [31].

VERA-CS generates sub pin level power profiles. While computationally expensive, this level of detail automatically creates a power history specific to each pin which can then be used for fuel performance analysis. This is superior to the less computational demanding approach of using few-group coarse-mesh nodal diffusion methods with pin powers reconstructed from the intra-nodal flux distributions [32].

The version 3.6 of VERA-CS used by this work utilizes table lookups of BISON generated tables for fuel performance data (specifically temperature profiles) in place of coupled fuel performance calculations. While this table lookup reduces computational workload, from an accuracy standpoint it is less than ideal as the optimal configuration is to tightly couple fuel performance calculations with neutronics, thermal hydraulic, and isotopic depletion calculations [33]. Also, the exclusion of concurrent fuel performance 
calculations prevents the reporting of outputs exclusive to fuel performance codes such as GCPP. Furthermore within COBRA-TF, the gap conductance is treated as a constant both temporally and spatially and the fuel thermal conductivity is burnup independent.

Current development at CASL is incorporating a $1 \frac{1 / 2}{\mathrm{D}}$ version BISON in order to improve fuel performance modeling [33], in the interests of improving neutron cross section estimations as well as including dynamic gap conductance and burnup dependent fuel thermal conductivity. Various strategies for partitioning and streamlining of the workload are being investigated in order to maintain reasonable computational expense [33].

\subsection{BISON}

BISON is a finite element based multidimensional and multiphysics fuel performance code [10, 34]. BISON allows for steady state and transient simulation of single fuel rod. Thermodynamic, mechanical, and species diffusion equations are fully-coupled and solved in a fully implicit manner. BISON is being developed with INL’s Multiphysics Object-Oriented Simulation Environment (MOOSE).

MOOSE is built to handle the systems of nonlinear partial differential equations often encountered in nuclear design. MOOSE allows for the equations of interest to be solved in a fully coupled manner using the Jacobian-free Newton-Krylov method [4]. The physical models involved are all modularized so as to allow for a flexible framework wherein new physics models can be added and combined in novel ways. This modularized approach allows for any reasonable combination of modules to be constructed in a less cumbersome manner than traditional code development. MOOSE coupling has been particularly successful in coupling material science calculations involving the atomistic, mesoscale, and engineering scale of fuel performance $[35,36]$.

BISON may be thought of as a set of MOOSE modules specific to fuel performance. Modules available to the fuel include temperature, burnup, and porosity dependent material properties, fission product swelling and densification strains, thermal and irradiation creep, fracture, and fission gas production and release. Models for cladding materials include plasticity, thermal expansion, irradiation growth, hydrogen uptake and creep. BISON also has models for coolant channels, cladding oxidation, plenum gas behavior, gap heat transfer, and contact between fuel and cladding.

BISON supports a variety of meshes including, 2-D smeared mesh, 3-D meshes, and 3-D discrete pellet meshes. BISON utilizes more complex, mechanistic methods to model phenomena such as fission gas release and contact, contrary to less mechanistic approaches used in other fuels performance codes such as FRAPCON (interested readers are referred to the user manual for FRAPCON [1] and BISON [10], specifically for fission gas release and the fuel and cladding mechanical response). While this comes at higher computational costs, the resulting output distributions for UQ and SA are less nonlinear compared to the abrupt shifts common in the piecewise functions of FRAPCON, as evidenced by past SA results [28]. BISON's 2D axisymmetric mesh allows for relatively fast running simulations and is used in this work.

\subsection{CODE INCONSISTENCIES}

Due to differences in domain, methodologies, and specific material property calculations, model inconsistencies between BISON and VERA-CS are inevitable. The aim of this work is either to minimize differences to an acceptable level and/or establish them as negligible. When neither of these is possible, the difference is carefully documented and given plans for future work. 
In regards to the domain, VERA-CS can model entire assemblies and/or full cores. The thermal hydraulic models used account for cross flow between the sub-channels. BISON models single fuel rods. Typical BISON models use a single coolant channel module to calculate heat transfer from the rod to coolant. However, this work transfers outer cladding data from VERA-CS in order to supply BISON with spatially and temporally varying temperature and pressures boundary conditions at the outer cladding surface. Therefore, the inconsistency is avoided by having the outer radial boundary condition of the cladding supplied by the thermal hydraulic models of VERA-CS alone without any thermal hydraulic modeling from BISON.

The discrepancy of greatest concern between the two codes is the fuel thermal conductivity. Past work $[19,28]$ has established it as the most dominant input in terms of impact on MFCT. The differences arise from VERA-CS currently not allowing burnup dependent fuel thermal conductivity. The exact empirical formulas used by BISON are available in COBRA-TF, however burnup data is currently not passed to the thermal conductivity routines. Fortunately this discrepancy will be remedied in the near future as CASL is currently developing burnup dependent thermal conductivity capabilities to be employed in later builds.

VERA-CS currently uses a constant gap conductance for all spatial and temporal locations. In reality, the gap conductance can vary greatly due to the differences in gap widths, gap gas temperature and composition, and interfacial pressures after contact. BISON currently models gap conductance with an extensive, mechanistic model. Past work established that when pins have open gaps, MFCT is sensitive to gap conductance parameters [28], hence the inconsistency may be of concern. This issue will also be addressed with later builds of VERA-CS which will include a dynamic gap conductance model.

VERA-CS has a highly advanced capability to model neutron transport while BISON is more rudimentary. Typically BISON uses a constant fast neutron flux to power density ratio, but also allows for a user defined average fast neutron flux power history. VERA-CS has the average fluxes available for 51 energy groups, however BISON is only interested in energies exceeding $1 \mathrm{MeV}$. The fast neutron flux for BISON is estimated according to the methodology presented in past work [37] and shown in Equation 1 ,

$$
\Phi_{E>1 M e V, i}=\sum_{j=L+1}^{L+N} \Phi_{j, i}+\frac{E_{L}-1 M e V}{E_{L}-E_{L+1}} \Phi_{L, i}
$$

where $\Phi_{j, i}$ is the flux for energy group $j$ at time $i$, and $L$ is index of the energy group which straddles $1 \mathrm{MeV}, N$ is the number of energy groups entirely above $1 \mathrm{MeV}$, and $E_{L}$ is the lower energy bound of group $L$. Essentially, Equation 1 performs a summation of the higher energy groups in addition to the portion of group $L$ above $1 \mathrm{MeV}$ determined via interpolation.

The average fast flux is then divided by the total core power to supply BISON with a core averaged (or assembly averaged in this case) fast flux to specific energy ratio. This method is still less than ideal as fast flux received by a given cladding is not a function alone of the fuel rod it encases, but also of the fuel rods in the local vicinity, as noted by Stimpson et al. [38]. A more ideal exchange of data would transfer the fast neutron flux averaged over each cladding surface. Unfortunately, this data is currently not available within the VERA-CS output files. Hence this level of coupling is only possible within the VERA-CS environment or with a custom version containing more in depth neutron flux output data. However, due to the shared enrichment and relatively low power variation within a single assembly case, the application of the average neutron fluxes to each individual rod is a fair approximation.

VERA-CS calculates isotopic depletion through the use of ORIGEN [39], while BISON uses TUBRNP or TRANSURANUS burnup [40]. The FOM of this work rely upon thermomechanics and thermal 
hydraulics, which are only slightly sensitive to isotopic depletion as a result of secondary effects related to changes in radial power peaking profiles. Therefore, it is safe to assume that this discrepancy is minor.

The aforementioned differences in fast neutron flux and isotopic depletion, as well as differences in calculating neutron cross sections create a difference in radial power profiles. Due to the buildup of plutonium near the outer radius of a fuel pellet, the local heat generation may be as much as three times that of average source term for a given axial location [10]. For this reason, the radial power profile has received particular attention from BISON developers, including a finer secondary grid for higher resolution of burnup and fission rates. As previously mentioned, VERA-CS contains far more in-depth neutronics calculations, however it is less adept at computing the temperature dependent cross sections.

Currently, this discrepancy is not resolvable as VERA-CS output files do not contain radial power profiles. The differences in the profile will be greatest near the outer radius edge, where the source terms are largest, thereby effecting properties such as outer fuel temperature and outer fuel burnup. However it will be far less impactful on properties calculated at locations other than the outer fuel radius (MFCT and MDNBR). The GCPP on the other hand may be effected by these discrepancies, as increased outer radius temperature affects gap gas temperature which in turn alters gas thermal conductivity. Thus the differences in radial profile merit further research to be conducted in later work.

While the fuel thermal conductivity, radial power profiles, and dynamic gap conductance discrepancies need be resolved in the future in the interests of improving LOTUS, it is essential to stress that the most critical data exchanged by the LOTUS scripts are the power and thermal hydraulic profiles. VERA-CS is at the cutting edge of coupled neutronics and subchannel thermal hydraulics reactor core simulations, and is capable of producing sub pin level power profiles of high fidelity, yet currently lacks various fuel performance analysis models. BISON has sophisticated fuel performance models, but is dependent upon idealizations or user supplied data for power history and thermal hydraulics data. The scope of this work is to create a more complete, but not yet all encompassing, framework reflective of true reactor physics. All discrepancies are minimized to the extent possible, documented, and highlight industry needs that may be resolved through future codes and/or new couplings.

\section{PROBLEM DESCRIPTION}

The computational resources required to perform full-core simulations with VERA-CS are not affordable for a Monte Carlo based sensitivity analysis. Therefore, the current work focuses on single assembly depletion cases lasting three fuel cycles for a total of four and a half years. Fuel design is a typical 17x17 fuel assembly with 264 fuel rods, 24 guide tubes, and 1 instrumentation tube. The assembly layout is shown in Figure 2, with the color blue denoting fuel rods, red indicating guide tubes for control rods, and the central yellow tile representing the guide/instrument tube. VERA-CS takes into account core and fuel assembly properties such as the upper and lower core plates, lower and upper assembly nozzles, and guide/instrument tubes. Eight spacer grids are included in the assembly to provide structural support as well as improve coolant mixing. The spacer grid form losses are a user input to the VERA-CS input file (see Table II). 


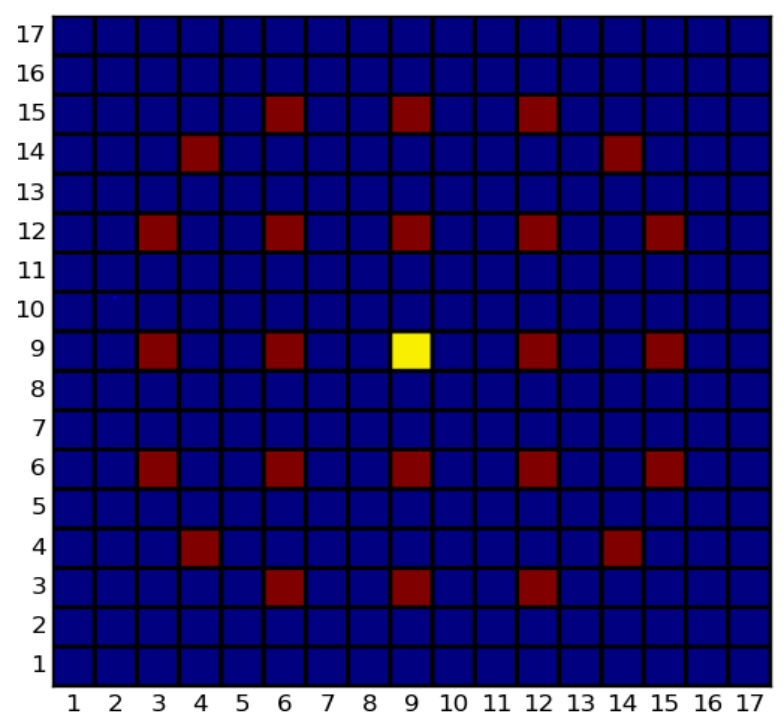

Figure 2. Assembly Layout with Colors Blue, Red, and Yellow Corresponding to Fuel Rods, Control Rods, and Guide/Instrument Tube Respectively

Each case was modeled without symmetry in order to get a better sense of the required computational load per assembly for a Monte Carlo sample. This gained experience will be of value when allocating resources for future work involving potentially hundreds of assemblies. Due to the problem having 1/8 symmetry, the maximum power locations are not limited to a single rod. Minor differences occur between theoretically identical pins as dictated by symmetry, due to the serendipitous nature of round off error. It was verified that all hot pin locations at the final state were in a consistent location according to $1 / 8$ symmetry for each instance of the Monte Carlo sampling.

Fuel performance is achieved via a full length rod with a smeared pellet mesh within BISON. The BISON model included solid mechanics, LWR specific heat transfer, burnup, contact, fuel relocation, fuel swelling, fission gas release, fuel and cladding creep, and cladding irradiative effects. The constant parameters supplied to both VERA-CS and BISON are given in Table I. Note that columns 3 and 4 indicate if the parameter of a given row is used by VERA-CS and/or BISON.

Table I. Design and simulation parameters for the model problem

\begin{tabular}{|l|c|c|c|}
\hline \multicolumn{1}{|c|}{ Parameter Name } & Parameter Value & VERA-CS & BISON \\
\hline Fuel Height & $3.657 \mathrm{~m}$ & $\mathrm{X}$ & $\mathrm{X}$ \\
\hline Pin Pitch & $1.26 \mathrm{~cm}$ & $\mathrm{X}$ & $\mathrm{X}$ \\
\hline Plenum Height & $16 \mathrm{~cm}$ & $\mathrm{X}$ & $\mathrm{X}$ \\
\hline Clad Top and Bottom Thickness & $1.67 \mathrm{~cm}$ & $\mathrm{X}$ & $\mathrm{X}$ \\
\hline Fuel Material & Uranium Dioxide & $\mathrm{X}$ & $\mathrm{X}$ \\
\hline Fill Gas Type & Helium & $\mathrm{X}$ & $\mathrm{X}$ \\
\hline Cladding Material & Zircaloy 4 & $\mathrm{X}$ & $\mathrm{X}$ \\
\hline Boron & $1640 \mathrm{ppm}$ & $\mathrm{X}$ & \\
\hline Plate Material & Stainless Steel & $\mathrm{X}$ & \\
\hline Plate Thickness (Lower/Upper) & $5.0 / 7.6$ & $\mathrm{X}$ & \\
\hline Plate Volume Fraction & 0.5 & $\mathrm{X}$ & \\
\hline Spacer Material (Middle/End) & $\mathrm{Inconel} /$ Zircaloy 4 & $\mathrm{X}$ & \\
\hline Spacer Height (Middle/End) & $3.810 \mathrm{~cm} / 3.866 \mathrm{~cm}$ & $\mathrm{X}$ & \\
\hline Spacer Mass (Middle/End) & $875 \mathrm{~g} / 1017 \mathrm{~g}$ & $\mathrm{X}$ & \\
\hline
\end{tabular}




\begin{tabular}{|c|c|c|c|}
\hline Nozzle Material & Stainless Steel & $\mathrm{X}$ & \\
\hline Nozzle Height (Lower/Upper) & $6.053 \mathrm{~cm} / 8.827 \mathrm{~cm}$ & $\mathrm{X}$ & \\
\hline Nozzle Mass & $6250 \mathrm{~g}$ & $\mathrm{X}$ & \\
\hline Pellet Height & $0.983 \mathrm{~cm}$ & & $\mathrm{X}$ \\
\hline Number of Pellets & 372 & & $\mathrm{X}$ \\
\hline Height of Pellet Dish & $0.0335 \mathrm{~cm}$ & & $\mathrm{X}$ \\
\hline Pellet End-Dish Shoulder Width & $0.0665 \mathrm{~cm}$ & & $\mathrm{X}$ \\
\hline Clad Fuel Bottom Gap & $0.001 \mathrm{~cm}$ & & $\mathrm{X}$ \\
\hline
\end{tabular}

\section{ANALYSIS METHODOLOGY}

\subsection{Figures of Merit}

The figures of merit (FOM) for this study are the minimum departure from nucleate boiling ratio (MDNBR), maximum fuel centerline temperature (MFCT), and gap conductance at peak power (GCPP).

The MDNBR is a thermal hydraulic variable defined as the ratio of the critical heat flux to a surface heat flux. This is expressed mathematically in Equation 2,

$$
M D N B R=\min \left(\frac{q_{\text {crit }}}{q}\right)
$$

where $q$ and $q_{\text {crit }}$ refer to the heat flux and critical heat flux respectively, and the $\min ()$ operator refers to the minimum across all spatial locations for a given time. An MDNBR equal to or less than unity indicates an undesirable boiling regime shift from nucleate boiling to transitional boiling, while MDNBR greater than one indicates the critical heat flux has not been reached. MDNBR is valuable from both a core performance and safety perspective. The MDNBR is not relevant to LOCA cases where convective boiling in the primary coolant loop is eclipsed by the effects of flash boiling.

The MFCT is a fuel performance metric which is simply the highest temperature calculated along the centerline of the fuel at a given time. It's worth noting that the MFCT may be different from the maximum fuel temperature of the entire rod, since radial power profiles can cause the maximum to exist between the center and outer radius. However, this is only encountered in the transient Reactivity Insertion Accidents (RIA), where fission rates are more extreme. In the standard operating conditions of this work, the maximum fuel temperature always resides on the center line, and thus MFCT represents the true maximum as well. Excessive temperatures are undesirable due to fuel cracking, and in extreme cases, fuel melting. MFCT is indirectly related to LOCA as it is closely related to the stored rod internal energy, an important initial condition to LOCA cases. MFCT was selected over stored rod internal energy due to it being more common in UQ/SA fuel performance studies.

The GCPP is a fuel performance metric which reports the thermal conductance of the gas filled gap between fuel and cladding at the location where peak power occurs for a given fuel rod. The gap conductance can be expressed as the summation of three separate terms shown in Equation 3,

$$
h_{\text {total }}=h_{\text {gas }}+h_{\text {solid }}+h_{\text {rad }}
$$

where $h_{\text {total }}$ is the total gap conductance, $h_{\text {gas }}$ accounts for gas thermal conductivity, $h_{\text {solid }}$ models the interfacial effects between cladding and fuel, and $h_{\text {rad }}$ represents radiative effects. Unlike MDNBR and MFCT which are predominantly linear, GCPP can, depending of the fuel performance model and fuel burnup, exhibit complex nonlinear interaction. 
GCPP is of particular interest to LOCA cases since it is impactful on safety criteria such as peak cladding temperature. Unfortunately, data transfer of gap conductance to SA codes specializing in LOCA entails transferring gap conductance data at all axial locations. This set of gap conductance data will differ slightly to the GCPP which is from a single higher power location. However, GCPP is still similar in behavior to the gap conductance at other all other locations. Thus UQ and SA studies are helpful in elucidating the nature of gap conductance uncertainty at any arbitrary location.

\subsection{Perturbed Inputs}

The perturbed inputs and their respective ranges are included in Table II. Note that an X in columns 4 and 5 indicate if VERA-CS and/or BISON use the input corresponding to a given row. Uncertainty ranges were obtained from [24] for thermal hydraulic parameters, [19, 21] for fuel performance, [22] for properties specific to fission gas release, and [41] for emissivities.

The * indicates that an input is not directly inputted into BISON, but rather directly affects an output from VERA-CS which is then treated as a BISON input. Specifically, the assembly power input directly affects power history supplied to VERA-CS. Similarly, the inlet temperature and outlet pressure have strong influence on the supplied temperature and pressure outer cladding boundary conditions used by BISON. Inputs which do not have a nominal value listed (such as fuel thermal conductivity), vary throughout the fuel cycle, and are thus biased by a consistent, specified amount (i.e. 10\%) throughout the simulation.

Table II. List of common uncertain parameters with their uncertainty range

\begin{tabular}{|l|c|c|c|c|}
\hline \multicolumn{1}{|c|}{ Input Name } & Nominal Value & Range(+/-) & VERA-CS & BISON \\
\hline Fuel Radius & $0.4096 \mathrm{~cm}$ & $0.001 \mathrm{~cm}$ & $\mathrm{X}$ & $\mathrm{X}$ \\
\hline Clad Inner Radius & $0.418 \mathrm{~cm}$ & $0.002 \mathrm{~cm}$ & $\mathrm{X}$ & $\mathrm{X}$ \\
\hline Clad Outer Radius & $0.475 \mathrm{~cm}$ & $0.002 \mathrm{~cm}$ & $\mathrm{X}$ & $\mathrm{X}$ \\
\hline Fuel Density (\% Theoretical) & $94.5 \%$ & $1.6 \%$ & $\mathrm{X}$ & $\mathrm{X}$ \\
\hline Fuel Enrichment (\% Weight) & $4.8 \%$ & $0.003 \%$ & & $*$ \\
\hline $\begin{array}{l}\text { Assembly Power } \\
\text { Cycle 1 }\end{array}$ & $17.674 \mathrm{MW}$ & $2 \%$ & $\mathrm{X}$ & \\
$\begin{array}{l}\text { Cycle 2 } \\
\text { Cycle 3 }\end{array}$ & $13.256 \mathrm{MW}$ & & & $*$ \\
\hline Inlet Temperature & $8.837 \mathrm{MW}$ & & $\mathrm{X}$ & $*$ \\
\hline Outlet Pressure & $554 .{ }^{\circ} \mathrm{F}\left(290.5{ }^{\circ} \mathrm{C}\right)$ & $3.0^{\circ} \mathrm{F}\left(1.667^{\circ} \mathrm{C}\right)$ & $\mathrm{X}$ & \\
\hline Direct Mod. Heating Fraction & $15.513 \mathrm{MPa}$ & $2 \%$ & $\mathrm{X}$ & \\
\hline Rated Flow & 0.02 & $10 \%$ & $\mathrm{X}$ & \\
& $3.410 \mathrm{Mlbs} / \mathrm{hr}$ & $2.0 \%$ & $\mathrm{X}$ & \\
\hline Mid Spacer Grid Form Loss & 0.9070 & $20 \%$ & $\mathrm{X}$ & \\
\hline End Spacer Grid Form Loss & 0.9065 & $20 \%$ & $\mathrm{X}$ & \\
\hline Void Drift Coefficient & 1.4 & $10 \%$ & & $\mathrm{X}$ \\
\hline Turbulent Mixing Coefficient & 0.005 & $10 \%$ & $\mathrm{X}$ \\
\hline Plenum Fill Gas Pressure & $2.0 \mathrm{MPa}$ & $0.08 \mathrm{MPa}$ & & $\mathrm{X}$ \\
\hline Fuel Surface Roughness & $2.0 \mu \mathrm{m}$ & $0.0333 \mu \mathrm{m}$ & & $\mathrm{X}$ \\
\hline Clad Surface Roughness & $1.0 \mu \mathrm{m}$ & $0.02 \mu \mathrm{m}$ & & $\mathrm{X}$ \\
\hline Fuel Surface Emissivity & 0.8 & $6.8 \%$ & & \\
\hline Clad Surface Emissivity & 0.325 & 0.1 & & \\
\hline Fuel Grain Radius & - & $60 \%$ & & \\
\hline Intra-Granular Diffusion & - & 0.1 to $10^{\dagger}$ & & \\
Coefficient & & & & \\
\hline
\end{tabular}




\begin{tabular}{|c|c|c|c|}
\hline $\begin{array}{l}\text { Grain-Boundary Diffusion } \\
\text { Coefficient }\end{array}$ & - & 0.1 to $10^{\dagger}$ & $\mathrm{X}$ \\
\hline $\begin{array}{l}\text { Intra-Granular Resolution } \\
\text { Coefficient }\end{array}$ & - & 0.1 to $10^{\dagger}$ & $\mathrm{X}$ \\
\hline $\begin{array}{l}\text { Fission Gas Release } \\
\text { Temperature Scaling Factor }\end{array}$ & - & $5 \%$ & $\mathrm{X}$ \\
\hline Cladding Creep & - & $30 \%$ & $\mathrm{X}$ \\
\hline Cladding Oxidation & - & $10 \%$ & $\mathrm{X}$ \\
\hline $\begin{array}{l}\text { Gas Gap Thermal } \\
\text { Conductivity }\end{array}$ & - & $5 \%$ & $\mathrm{X}$ \\
\hline Fuel Thermal Conductivity & - & $10 \%$ & $\mathrm{X}$ \\
\hline Clad Thermal Conductivity & - & $31.25 \%$ & $\mathrm{X}$ \\
\hline Fuel Thermal Expansion & $10 * 10^{-6} \mathrm{~K}^{-1}$ & $15 \%$ & $\mathrm{X}$ \\
\hline Clad Thermal Expansion & $5 * 10^{-6} \mathrm{~K}^{-1}$ & $15 \%$ & $\mathrm{X}$ \\
\hline
\end{tabular}

Note: * indicates an input which strongly effects a BISON input, but is not directly inputted.

$\dagger$ denotes a logarithmic range

All input distributions are a truncated normal distribution of two standard deviations with the exceptions of the intra-granular diffusion coefficient, grain boundary diffusion coefficient, and intra-granular diffusion coefficient. As denoted by ${ }^{\dagger}$, these uncertainties are logarithmic, and vary by a factor of 100 ( 0.1 to 10 ) [22]. These scaling factors in the LOTUS scripts are of the form $10^{0.5 \sigma}$, where $\sigma$ is a random variable selected from a truncated random distribution of two standard deviations, with a mean of zero and a standard deviation of unity.

It is worth noting that many of the inputs, such as cladding inner and outer radius are highly correlated. Plans for future work hope to properly sample the inputs with the appropriate correlations. However, this is a large undertaking and is outside the scope of this work. The assumption of independent inputs is not uncommon in UQ/SA literature [19] and serves as a starting point in understanding the uncertainty of a given output.

Lastly it must be acknowledged that many fuel performance inputs, such as fuel radius, should ideally be treated as separate for each pin. However, there are two disadvantages to this treatment. First, a smoothing effect occurs since each fuel assembly becomes a mix of the individually perturbed fuel rods, causing the mean value of any fuel property to remain relatively consistent between Monte Carlo perturbations. Secondly, treating 22 fuel performance inputs as unique to each of the 264 fuel rods results in 5808 separate inputs. While this large set of inputs is acceptable for UQ studies, such a vast number is unreasonable when conducting an effective SA study.

\subsection{Monte Carlo Sampling and Data Flow}

All UQ and SA metrics are obtained from a Monte Carlo sampling among the perturbed inputs listed in Table II. The single assembly case in conjunction with the high performance computing powers available at INL allows for a Monte Carlo sampling of VERA-CS followed by a direct data exchange for power histories and thermal hydraulic profiles to BISON for the hottest pin (the highest power pin at the end of the fuel cycles). The precise methodology is shown in Figure 3. 


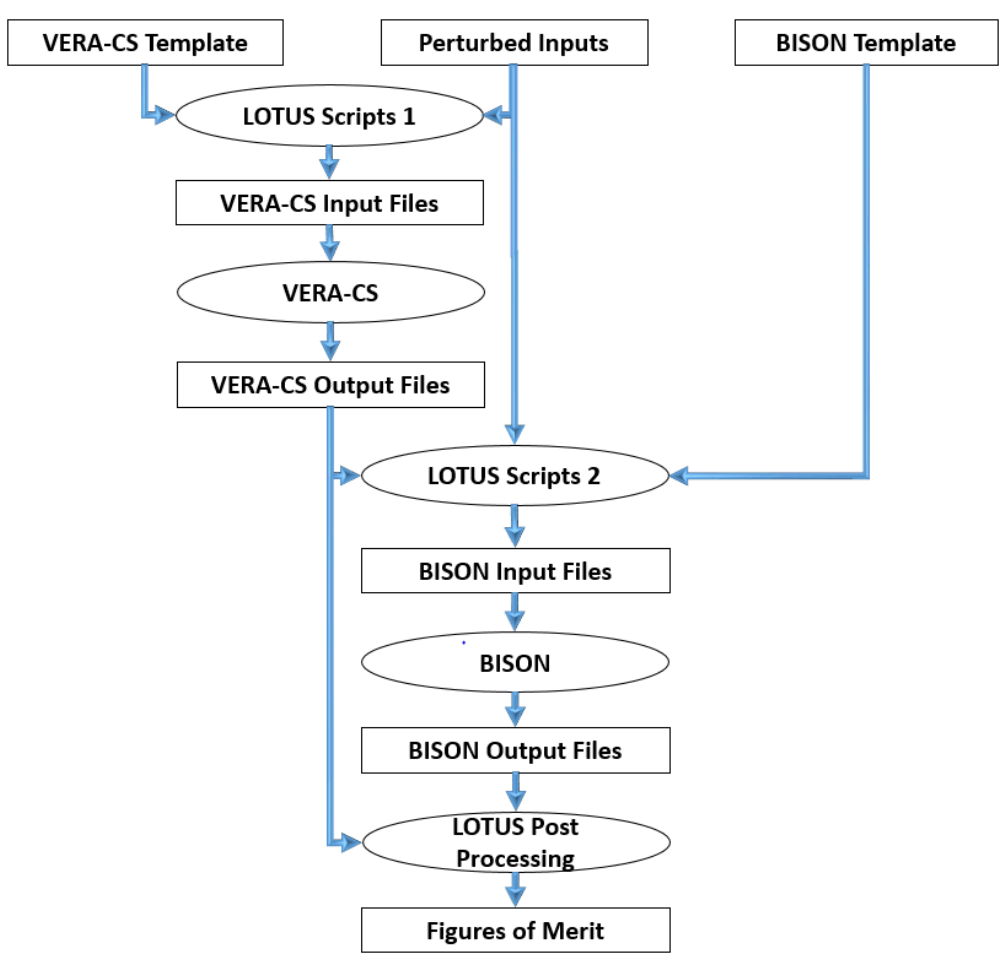

Figure 3. Data Flow for Monte Carlo Sampling

In instances where the computational expense of a code does not permit a large sample size, a smaller sample must be used, from which PDF of the desired outputs are estimated. The more computationally inexpensive codes then perform a larger resample from the generated PDF. Fortunately at INL, the high performance computer (HPC) resources negate the need for PDF generation and resampling and permit a more ideal direct data connection.

While past work with FRAPCON allowed for a run of every pin in the assembly due to FRAPCON run times of approximately two seconds [28], the current work is hindered by BISON runtimes of approximately two hours. Fortunately, the fuel performance FOM of MFCT and GCPP have maximums which reside on the highest power pin for all cases observed in this work. Since the highest power pin position varies throughout the fuel lifecycle, the pin of interest was selected on the basis of highest power pin at the end of the fuel lifecycle. The data for this pin was passed to VERA-CS for all states, despite it not necessarily being the hottest pin for a given time. While this selection method is less than ideal, it assures that all data analysis is applied to a consistent pin location. The data transfer from VERA-CS includes cladding and fuel dimension, outer cladding temperatures and pressures, pin power histories including axial power shapes, and assembly averaged fast neutron flux history.

\subsection{Uncertainty Quantification}

LOTUS allows for uncertainty quantification (UQ) and sensitivity analysis (SA) of figures of merit (FOM) resulting from calculations in a multiphysics environment. The uncertainty within LOTUS is quantified via a 95/95 confidence interval given by Equations 4 and 5,

$$
\begin{gathered}
Y_{95 / 95}=\mu_{95 \%} \pm 1.96 * S E_{Q 95 \%} \\
S E_{Q 95 \%}=2.11 * S E_{M}
\end{gathered}
$$


where $Y_{95 / 95}$ is the $95^{\text {th }}$ percentile value for a given output, and $S E_{Q 95 \%}$ is the standard error associated with said value [42]. Put simply, the 95/95 confidence interval is a bound which contains 95 percent of the total population with 95 percent certainty. It should be noted that estimation of the standard error assumes a normal profile, which is not strictly true in the case of GCPP. Research into Monte Carlo based UQ for arbitrary probability distributions will be a focus of future work.

\subsection{Sensitivity Analysis}

Unlike UQ which contains a relatively linear set of common methodologies, SA methods contain an ever expanding list of measures. The methods vary in computational cost and in their ability to elucidate complex behavior. The SA measures of this work include two regression based measures (Pearson and Spearman), one variance based (Sobol), and three moment independent measures (Borogonov Delta measure and $\mathrm{L}_{2}$ and $\mathrm{L}_{\infty}$ variations).

\subsubsection{Regression Based Sensitivity Measures}

Pearson correlation coefficients are a regression based measure. A useful visualization of regression methods is the passing of a straight line through a scatterplot of data. If all the data lies perfectly on the fitted line, the Pearson measure is unity. If all straight lines have no bearing on the data (excluding lines with zero slope), then the measure is zero. Pearson correlation coefficients can be found by Equation 6 ,

$$
R_{i}^{2}=\frac{\operatorname{cov}\left(X_{i}, Y\right)^{2}}{\operatorname{var}\left(X_{i}\right) \operatorname{var}(Y)}
$$

Among Monte Carlo methods, Pearson measure requires smaller sample sizes. As opposed to more advanced methods, the Pearson measure does not require a computationally expensive Monte Carlo double loop sampling, or data partitioning which generates additional noise in the data.

While the method is capable of detecting linear trends, strong nonlinear trends do not manifest. For instance, the simple relation $Y=|X|$ yields an $R^{2}$ which approaches zero as the sample size of $X$ approaches infinity. Fortunately the linearity of the system may be tested a posteriori via the summation $\sum_{i=1}^{N} R_{i}^{2}$, where $N$ is the number of inputs. Summations equal to unity are fully linear systems, while summations far from unity contains large nonlinearities.

This testing is of great utility to decision makers. If a low summation is found then the decision maker knows a higher order method, and thus large sample size, is needed. However, if the sum is nearly unity, a large sample for higher order methods is unnecessary and Pearson measures may become the highest fidelity measure due to their low noise.

A natural extension of the Pearson measure is the Spearman correlation coefficient. Spearman differs in that it evaluates the rankings of inputs and outputs as opposed to the actual values. This can be expressed mathematically in Equation 7,

$$
\rho_{i}^{2}=\frac{\operatorname{cov}\left(X_{i}^{r n k}, Y^{r n k}\right)^{2}}{\operatorname{var}\left(X_{i}^{r n k}\right) \operatorname{var}\left(Y^{r n k}\right)}
$$

where $\rho_{i}^{2}$ is the Spearman correlation coefficient, and $X_{i}^{r n k}$ and $Y^{r n k}$ are the rankings of an arbitrary input and output. Spearman is advantageous over Pearson in that it is superior in detecting nonlinearities, 
as long as the relations are monotonic. However, if the trends are non-monotonic, then Spearman may perform poorly.

\subsubsection{Variance Based Sensitivity Measures}

Sobol indices are a variance based measure, which is to say they quantify the impact a given input has on the variance of an output. The indices are a function of the variance of the expected value of the output conditional upon the fixing of an input. The indices are normalized by dividing the aforementioned variance by the total variance of the output. This is expressed mathematically in Equation 8,

$$
S_{i}=\frac{\operatorname{var}\left(E\left(Y \mid X_{i}\right)\right)}{\operatorname{var}(Y)}
$$

If the output is predominantly a function of said input, then the expected value of the conditional output will vary greatly, yielding a large variance in the numerator of Equation 8. However, if the output is a relatively weak function, then the expected value of the conditional output will be nearly the expected value of the unconditional output, with zero to very low variance.

The main benefit to variance based methods over regression based methods is the ability to detect nonlinear effects that do not include interactions between inputs. Sobol indices are very adept at analyzing an additive systems (i.e. $Y=f\left(X_{1}\right)+g\left(X_{2}\right)$ ). This is true even if the individual functions are nonlinear. However, Sobol indices become ineffective in detecting the interactions between inputs (i.e. $\left.Y=X_{1} X_{2}\right)$.

Sobol indices ideally require a double loop Monte Carlo sampling. Fortunately Plischke partitioning [43] allows for the partitioning of data from a large single loop Monte Carlo sampling, as shown in Equation 9,

$$
\widehat{S}_{i}=\frac{\frac{1}{N} \sum_{j=1}^{M} N_{j} \operatorname{var}\left(Y \mid X_{i} \in x_{i, j}\right)}{\operatorname{var}(Y)}
$$

where $\widehat{S}_{i}$ is the approximation of the Sobol indices, $N$ is the total sample size, $M$ is the number of partitions, $N_{j}$ is the number instances in partition $j$ and $Y \mid X_{i} \in x_{i, j}$ represents a sample of $Y$ given that $X_{i}$ is one of the instances of $X_{i}$ in partition $j . x_{i, j}$ is formed by first ranking all instances of $X_{i}$, placing them in order, and then partitioning $M$ roughly equal spaced groups. The number of partitions is selected based upon an SA library available in Python [44] and shown in Equation 10,

$$
M=\min \left(\uparrow\left(N^{\left(\frac{2}{7-\tanh \left(\frac{1500-N}{500}\right)}\right)}\right), 48\right)
$$

where $\uparrow()$ operator rounds real numbers up to the next integer. This process allows for estimating Sobol indices with a purely random distribution. A bootstrapping method with 10 resamples is also employed in the interests of reducing noise caused by the Plischke partitioning [45].

\subsubsection{Density Based Sensitivity Measures}

Density based moments are independent of the moments of the data (i.e. mean and variance) [29]. Delta measures seek to quantify changes between the probability distribution functions (PDF) of the output and the PDF of the output conditional upon the fixing of a given input. The most widely used Delta measure established by Borogonov is the $\mathrm{L}_{1}$ norm between said densities as shown in Equation 11, 


$$
\delta_{i}^{1}=\frac{1}{2} E_{X_{i}}\left(s_{i}^{1}(Y)\right)
$$

where $\delta_{i}^{1}$ is the $\mathrm{L}_{1}$ norm Delta measure, $E_{X_{i}}()$ is the expected value operator when input $X_{i}$ is varied, and $s_{i}^{1}()$ is given by Equation 12,

$$
s_{i}^{1}\left(X_{i}\right)=\int_{-\infty}^{\infty}\left|f_{Y}(y)-f_{Y \mid X_{i}}(y)\right| d y
$$

where $f_{Y}$ is the unconditional probability distribution function, and $f_{Y \mid X_{i}}$ is the probability density conditional upon fixing input $X_{i}$. Thus if the density is affected in any way by the fixing of an input, the Delta measure will register the change. Furthermore the summation of all $\delta_{i}^{1}$ is equal to unity if the inputs are separable, which is to say the density shifts of any given input is independent of the fixing of additional inputs. Recently the Delta measure has been extended to include $\mathrm{L}_{2}$ and $\mathrm{L}_{\infty}$ [30]. The $\mathrm{L}_{2}$ norm is given by Equation 13,

$$
\delta_{i}^{2}=E_{X_{i}}\left(s_{i}^{2}(Y)\right)
$$

where $\delta_{i}^{2}$ is the $\mathrm{L}_{2}$ norm Delta measure and $s_{i}^{2}()$ is given by Equation 14,

$$
s_{i}^{2}\left(X_{i}\right)=\sqrt{\int_{-\infty}^{\infty}\left(f_{Y}(y)-f_{Y \mid X_{i}}(y)\right)^{2}}
$$

Unlike $\mathrm{L}_{1}$ norms which given equal weight between all differences in densities, $\mathrm{L}^{2}$ norms give greater weight to larger differences. The summation of all the $\delta_{i}^{2}$ for each input does not have any significant meaning. The infinity norm is a function of the largest density difference alone. The infinity norm is given by Equation 15,

$$
\delta_{i}^{\infty}=E_{X_{i}}\left(s_{i}^{\infty}(Y)\right)
$$

where $\delta_{i}^{\infty}$ is the $\mathrm{L}_{\infty}$ norm Delta measure and $s_{i}^{\infty}$ is given by Equation 16 ,

$$
s_{i}^{\infty}\left(X_{i}\right)=\max \left(f_{Y}(y)-f_{Y \mid X_{i}}(y)\right)
$$

Like the $\mathrm{L}_{2}$ norm Delta measure, the summation of $\mathrm{L}_{\infty}$ norm Delta measures has no significant meaning. To make $\delta_{i}^{2}$ and $\delta_{i}^{\infty}$ comparable between two different FOM, each FOM is mapped to a domain from zero to unity. Furthermore, $\delta_{i}^{2}$ and $\delta_{i}^{\infty}$ are also normalized in the interests of being more comparable to the other SA measures of this work. Note that the normalization does not affect rankings when comparing two inputs.

Like variance based methods, density based measures also ideally require a double Monte Carlo loop. This is remedied by using the Plischke partitioning [45] to group the outputs into sets of roughly equal size according to the ranking of a given input. The number of partitions is selected in the same manner as variance based measures (see section 4.5.2, Equation 10). Equations 11, 13, and 15 can be recast as Equation 17,

$$
\widehat{\delta_{l}^{p}}=\frac{1}{2 N} \sum_{j=1}^{m} N_{j} s_{i}^{p}\left(X_{i} \in x_{j}\right)
$$


where $N$ is the total sample size, $N_{j}$ is the size of partition $j, x_{j}$ are the instances of input $X_{i}$ residing in group $j$, and $s_{i}^{p}\left(X_{i} \in x_{j}\right)$ is L norm of order $p$ (i.e. $\left.1,2, \infty\right)$ between the unconditional density and density conditional upon $X_{i}$ residing within $x_{j}$ group. $s_{i}^{p}\left(X_{i} \in x_{j}\right)$ is expressed mathematically as Equation 18,

$$
s_{i}^{p}\left(X_{i} \in x_{i, j}\right)=\sqrt[p]{\int\left|\hat{f}_{Y}(y)-\hat{f}_{Y \mid X_{i} \in x_{i, j}}(y)\right|^{p} d y}
$$

Note that Equations 18 contain the terms $\hat{f}_{Y}$ and $\hat{f}_{Y \mid X_{i} \in x_{i, j}}$ which are the kernel density estimates of the unconditional and conditional PDF respectively given by Equation 19,

$$
\widehat{f_{y}}=\frac{1}{N \lambda} \sum_{i=1}^{N} K\left(\frac{y-y_{i}}{\lambda}\right)
$$

where $N$ is the sample size, $y_{i}$ is an instance of the random variable $Y, \lambda$ is the kernel bandwidth, and $K()$ is the kernel function. This work employs Gaussian kernels as shown in Equation 20 with bandwidth selection based on Botev's method [46].

$$
K(z)=\frac{1}{\sqrt{2 \pi}} e^{-\frac{z^{2}}{2}}
$$

A reflective boundary condition is enforced at the upper and lower bound which forces all integrations of the PDF to maintain unity regardless of bandwidth selection [46],

$$
\hat{f}_{y}^{R}=\frac{1}{N \lambda} \sum_{j=-R}^{R} \sum_{i=1}^{N} K\left(\frac{y-y_{i}-j * W}{\lambda}\right)
$$

where $\hat{f}_{y}^{R}$ is the kernel density estimate with reflective boundary conditions, $W$ is the width of the domain, and $R$ is the number of reflections ( $R=1$ is sufficient with the exception of cases with bandwidths near or greater than the domain width, i.e. near uniform distributions). The implementation of these kernel density estimates was achieved via the Python library PyQt-Fit [47].

\section{RESULTS}

\subsection{Uncertainty Quantification Results}

All UQ results follow the format of the $95^{\text {th }}$ percentile value and the $+/-$ range for a $95 \%$ confidence interval. This information is shown roughly every 50 days throughout the 3 fuel cycles which corresponds to four and a half years.

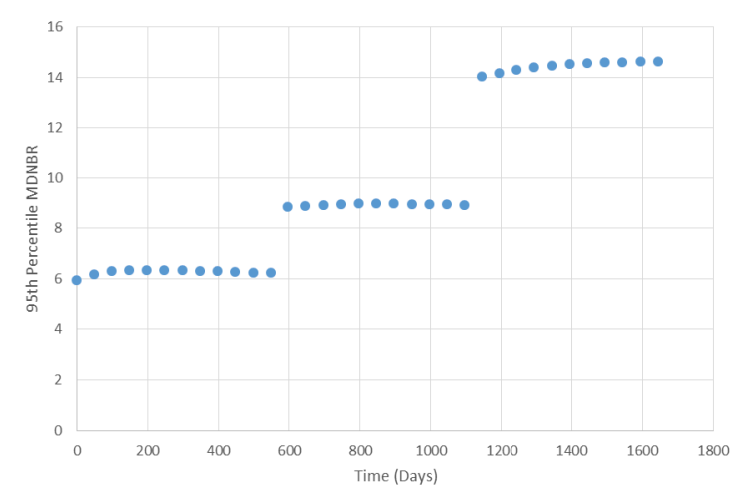

a)

b)

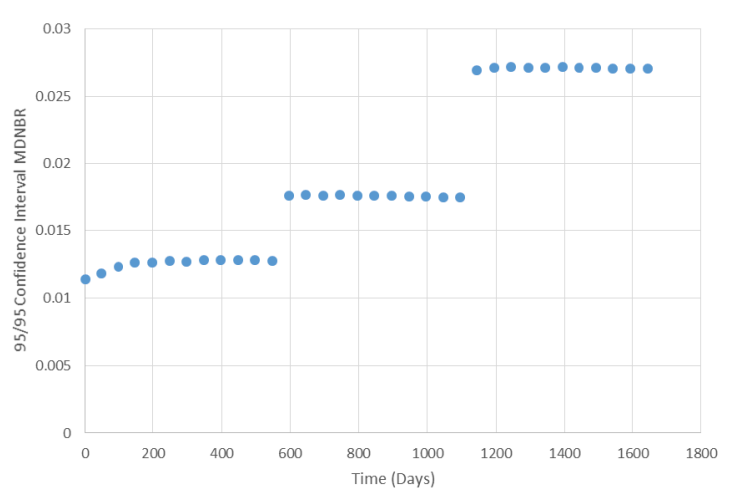


Figure 4. a) $95^{\text {th }}$ Percentile and b) $+/-95 \%$ Confidence Interval for MDNBR

UQ results for MDNBR are shown in Figure 4. The abrupt increases in MDNBR correspond to differences in core power among fuel cycles (see Table II). The confidence interval is nearly proportional to the $95^{\text {th }}$ percentile value and relatively small, never exceeding $0.21 \%$ of the $95^{\text {th }}$ percentile value. The MDNBR increases as power decreases for each fuel cycle due to the relation stated in Eq. 2. Simply put, as the core power decreases, the heat flux decreases proportionally, and thus the denominator of Eq. 2 decreases, causing an increase in MDNBR. Figure 4 indicates that the case under investigation in this work is not in proximity to a departure from nucleate boiling at any point in time. The aforementioned narrow confidence interval indicates the $95^{\text {th }}$ percentile value is of high fidelity.

a)

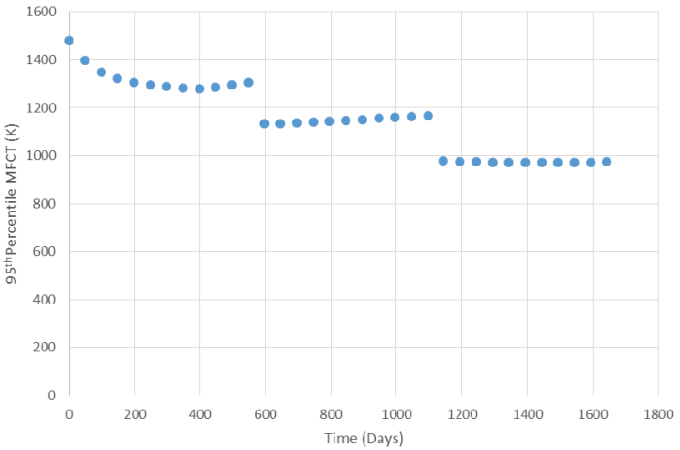

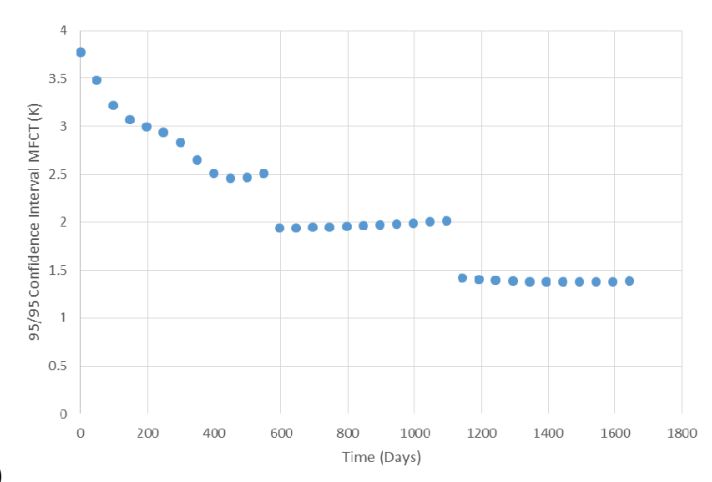

b)

Figure 5. a) $95^{\text {th }}$ Percentile and b) $+/-95 \%$ Confidence Interval for MFCT

UQ results for MFCT are shown in Figure 5. As before, the abrupt drops in MFCT coincide with the lowering of power between fuel cycles. The relative size of the confidence interval values is very similar to MDNBR, with the confidence interval values never greater than $0.26 \%$ of the $95^{\text {th }}$ percentile value. However, the relative error is significantly higher for the higher temperature states than proportionality to the $95^{\text {th }}$ percentile alone can account for. This is due to the added uncertainty from open gap conductance models in the earlier states (see section 5.2.2).

The MFCT initially decreases during cycle one due to the closing of the gap (see Figure 6), then slightly increases towards the end of cycle one and all of cycle two. This increase is likely attributable to a gradual decrease in fuel thermal conductivity due to fuel swelling, which increases porosity. The third cycle only slightly decreases for the majority of the fuel cycle, most likely due to the buildup of plutonium in the outer radius, leading to a decreased radial power peaking factor in the interior of the rod, causing a small decrease in MFCT. The final MFCT increase is likely due to irradiation creep reducing the interfacial pressure between fuel and cladding, thereby decreasing gap conductance and increasing MFCT slightly. 


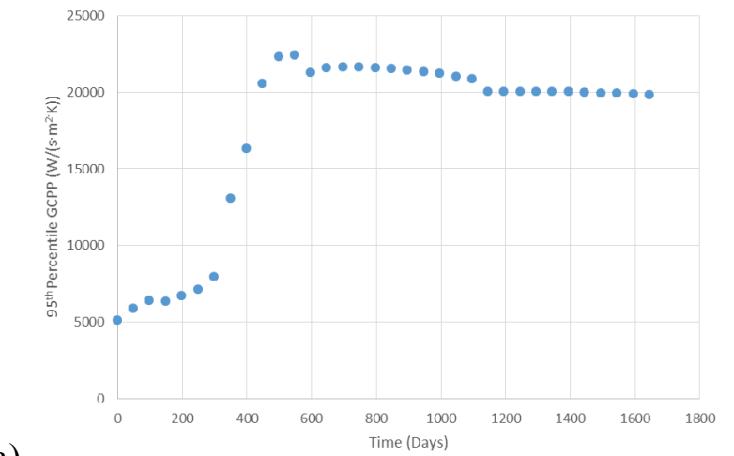

a)

Figure 6 a) $95^{\text {th }}$ Percentile and b)

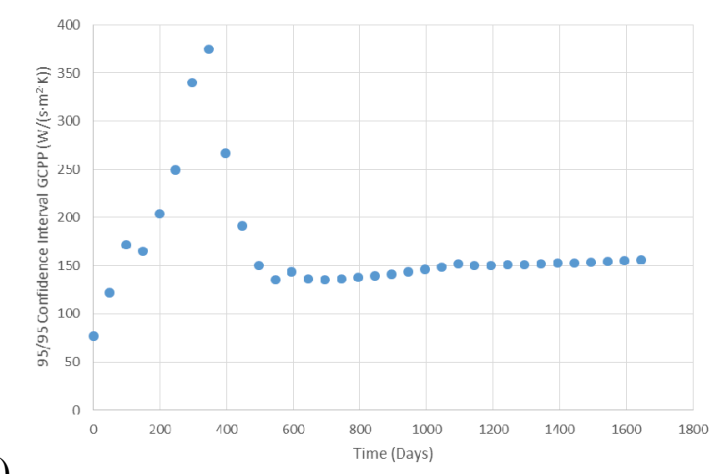

b)

b) $+/-95 \%$ Confidence Interval for GCPP

The UQ results for GCPP are shown in Figure 6. Figure 6a shows some minor drops for the changes in fuel cycles, but the dominant behavior is attributable to the closing of the gap. The initial increase after closure is likely due to increased interfacial pressure from swelling, after which cladding irradiation creep becomes more dominant causing a decrease in interfacial pressure. The closing of the gap results in a higher gap conductance and a shift in models which changes the associated uncertainty. The largest spike in uncertainty corresponds to a time in which a portion of the Monte Carlo sampled cases are in contact while the remainder retain an open gap. This stratifying of the sample increases the total variance, thus widening the $95 / 95$ confidence interval to as high as $4.2 \%$ of the $95^{\text {th }}$ percentile value.

\subsection{Sensitivity Analysis Results}

\subsubsection{Sensitivity Analysis of Minimum Departure from Nucleate Boiling Ratio}

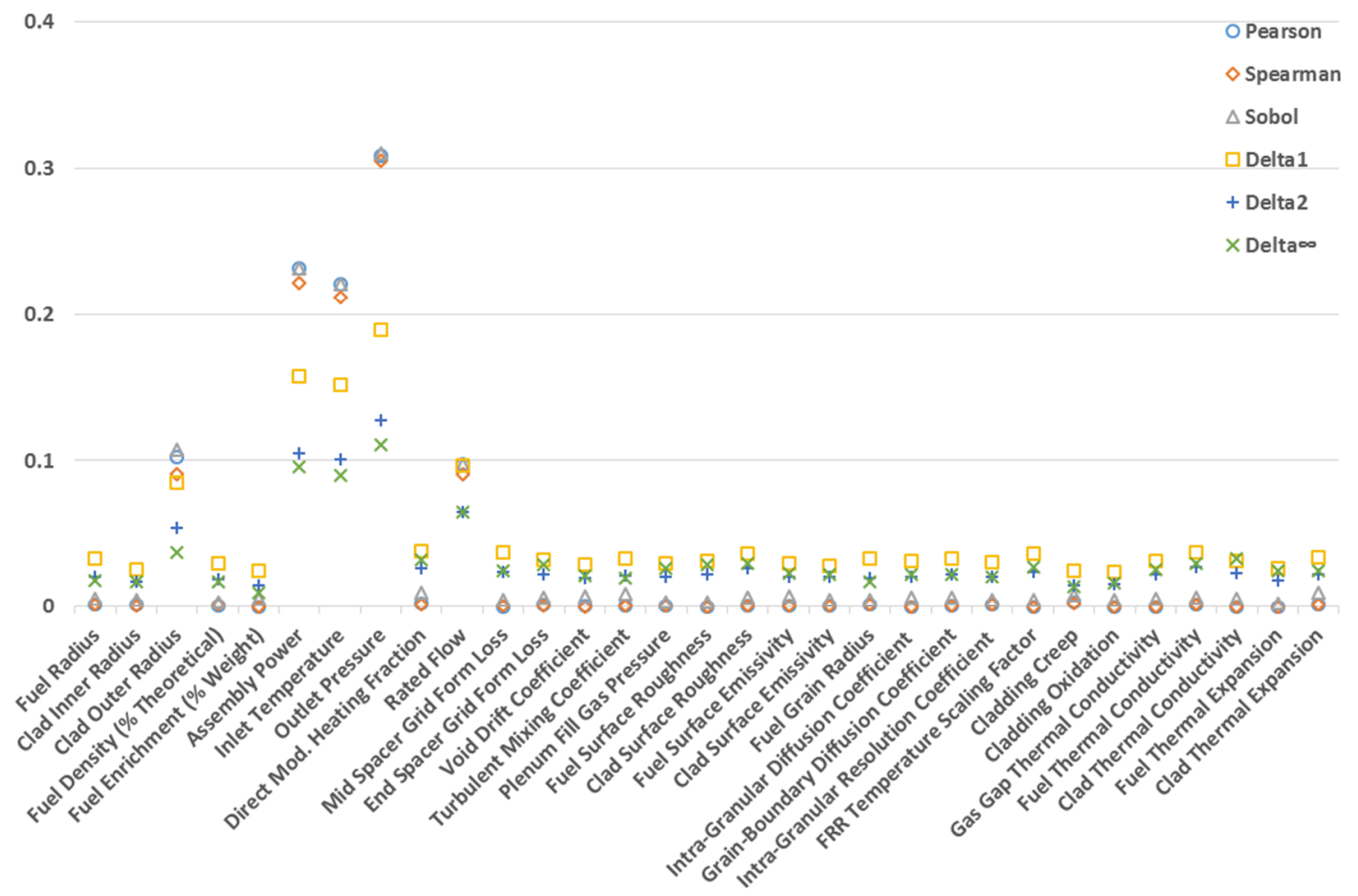

Figure 7. Sensitivity Analysis Measures for MDNBR at 1644 Days 
Results for MDNBR showed consistent rankings between all SA measures with negligible change in measures from state to state. Thus MDBNR analysis does not gain insight from computing the steady state solution to 34 states across three fuel cycles. However, benefits will be apparent in the UQ and SA results of the fuel performance FOM of MFCT and GCPP, which are far more sensitive to the cumulative effects of depletion. For the sake of brevity, only the final state is reported in Figure 7.

The summation of Pearson measures is 0.98 , indicating highly linear relations, thus all differences in methods will stem from noise in partitioning methods and kernel density estimations. Figure 7 indicates that outlet pressure is the most impactful, with inlet temperature and assembly power also playing significant roles, and notable impact from rated flow and clad outer radius. This indicates that if greater fidelity in MDNBR values is desired, the two primary areas of focus must be thermal hydraulic boundary conditions (inlet temperature and outlet pressure) and the operating power.

Not surprisingly, all inputs exclusive to fuel performance calculations have no impact on MDNBR. This is due to MDNBR being a thermal hydraulic FOM. In the current framework, the MDNBR reported is obtained directly from VERA-CS, completely independent of all BISON calculations.

\subsubsection{Sensitivity Analysis of Maximum Fuel Centerline Temperature}

The MFCT behaves predominantly linearly throughout the fuel lifecycle. The rankings among SA measures are consistent for each state. However significant shifts in SA measures do occur throughout the cycle. The shifts coincide with the onset of contact. Figures 8, 9, and 10 show SA measures for MFCT at the pre-burnup, open gap state, mixed open and closed gap, and predominantly closed gap states respectively.

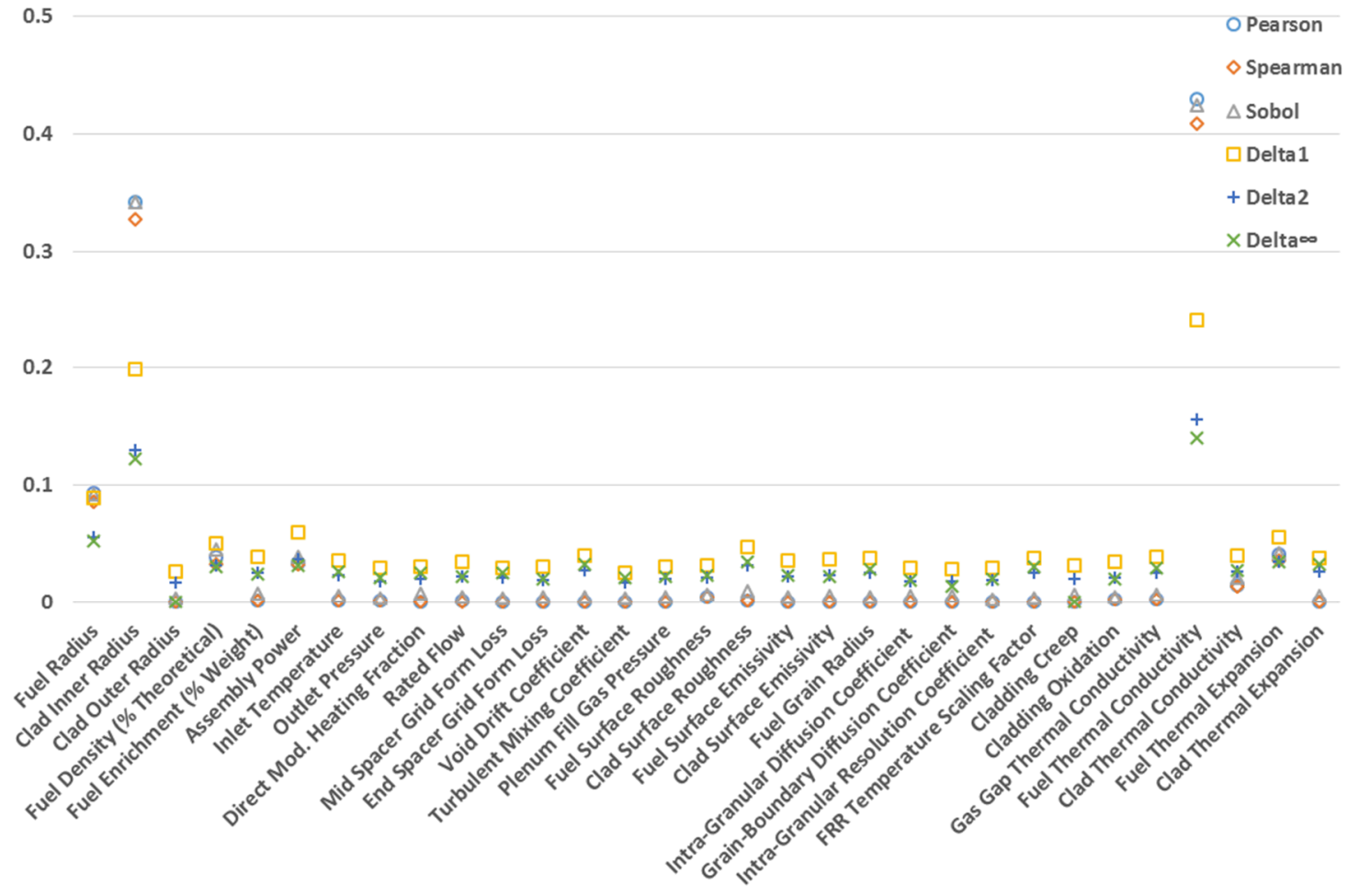

Figure 8. Sensitivity Analysis Measures for MFCT at 0 Days 


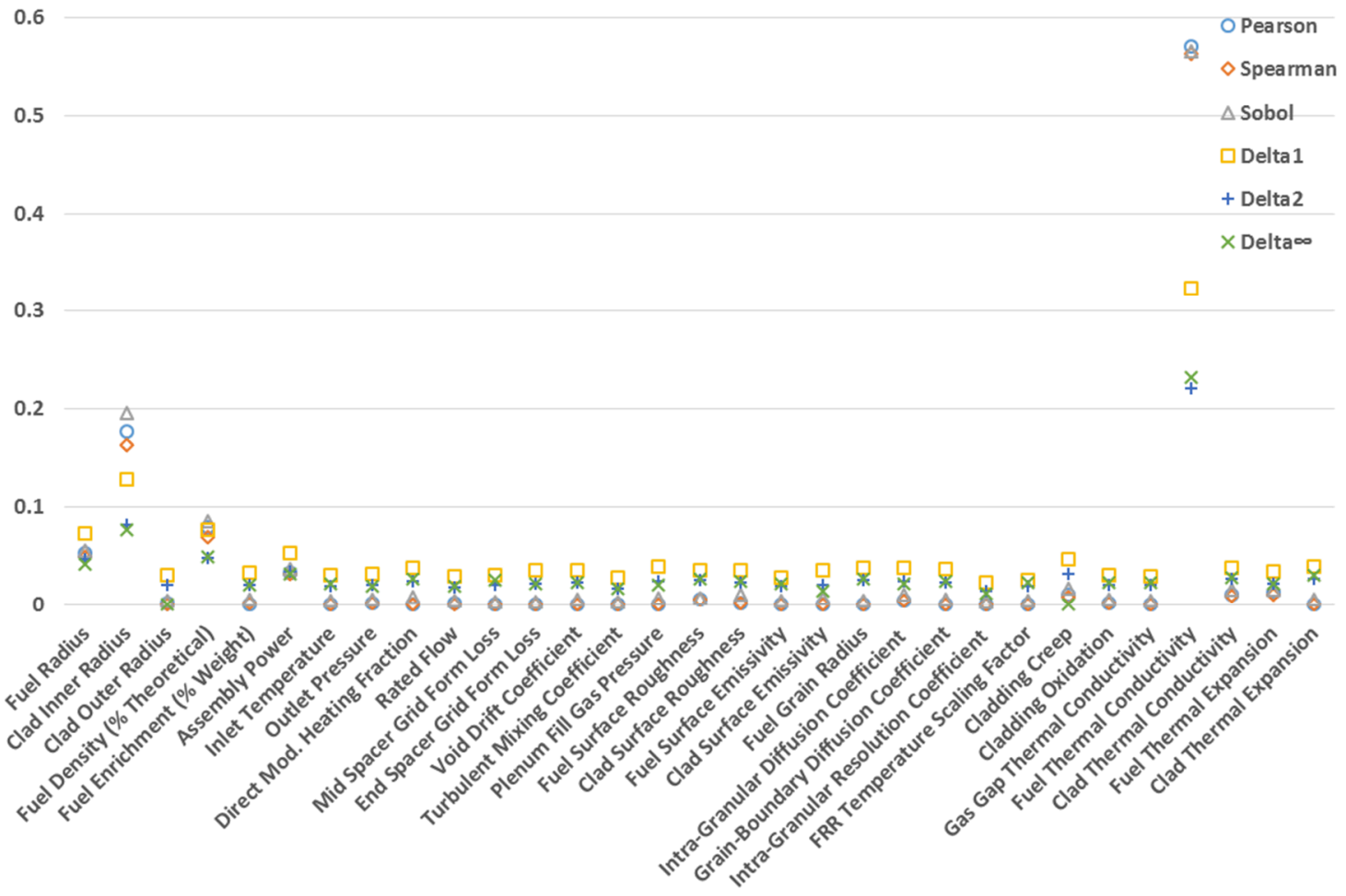

Figure 9. Sensitivity Analysis Measures for MFCT at 348 Days

The summation of Pearson measures for Figure 8 is 1.01, indicating a linear system, thus all differences in SA measures stem from noise in partitioning methods and kernel density estimation. Figure 8 shows that at the start of the fuel lifecycle, before burnup up has occurred, uncertainty primarily stems from fuel thermal conductivity due to its direct role in the gradients of temperature in the heat transfer calculation. Clad inner radius and fuel radius also contribute considerably due to their effects on gap width, which affects gap conductance. Note also that fuel thermal expansion has a slight impact due to its effect on gap width. Fuel density and core power also play a minor role due to their effect on burnup which affects fuel thermal conductivity. The SA measures remain relatively consistent between states and among each other in terms of rankings until the onset of contact, which is shown in Figure 9.

Figure 9 has a Pearson squared summation of 0.97 and shows a strong reduction in SA measures for gap dimensions in contrast to the pre-contact data of Figure 8. This confirms intuition, since at this time many of the cases have transitioned from open to closed gap, resulting in a severely decreased dependency on gap dimension. As time progresses, eventually all cases contain closed gaps as shown in Figure 10. 


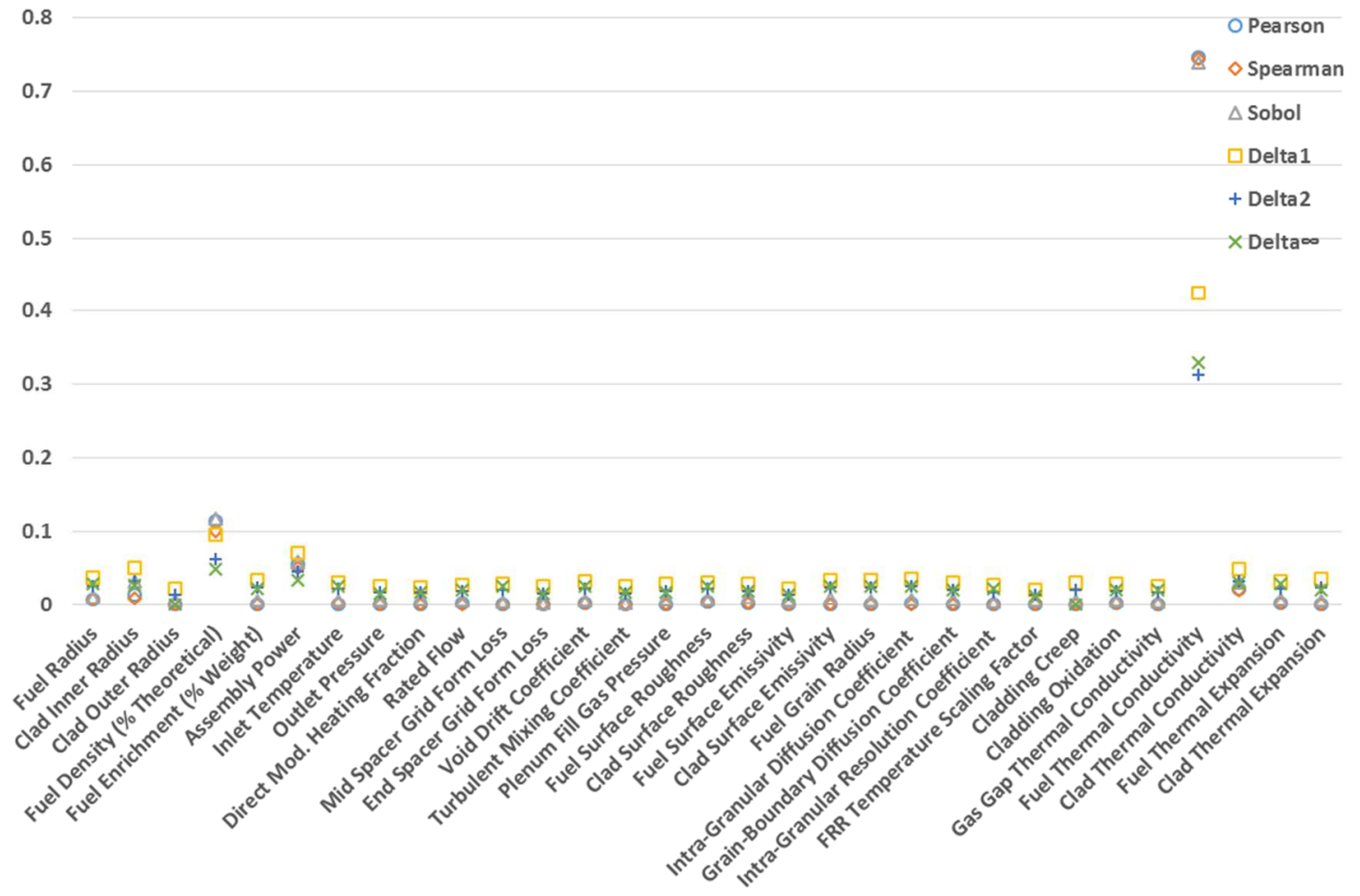

Figure 10. Sensitivity Analysis Measures for MFCT at 448 Days

Pearson squared summation for Figure 10 is 0.98 , once again indicating most effects are linear. Figure 10 shows that once most cases are in contact, nearly all uncertainty originates from fuel thermal conductivity with some effects from assembly power and fuel density due to their effect on burnup. This does not indicate that the MFCT is insensitive to assembly power, but rather that the MFCT uncertainty stemming from a 10\% uncertainty in fuel thermal conductivity dwarfs the MFCT uncertainty arising from a $2 \%$ uncertainty in core power (see Table II). Furthermore, the increase in SA measures on fuel thermal conductivity does not necessarily indicate an increased dependence of said input. Rather, it is more likely that the uncertainty stemming from gap width has ceased, causing a greater portion of the total uncertainty to originate from thermal conductivity.

Interestingly, at this state the fourth ranked input is clad thermal conductivity with a Pearson measure of 0.022 , further emphasizing the near complete dependence on specific material properties once the gap is closed. The remainder of the life cycle shows little change in SA measures.

\subsubsection{Sensitivity Analysis of Gap Conductance at Peak Power}

Unlike MDNBR and MFCT, GCPP allows a showcasing of the higher order methods due to the presence of nonlinearities. The exclusively open and closed gap states are predominantly linear, however the intermediate states contain substantial nonlinearities due to the shifting between open and closed gap models as well as complexities stemming from fission gas generation. SA measures for GCPP at the preburnup state and mixed open and closed gap state are shown in Figure 11 and 12. 

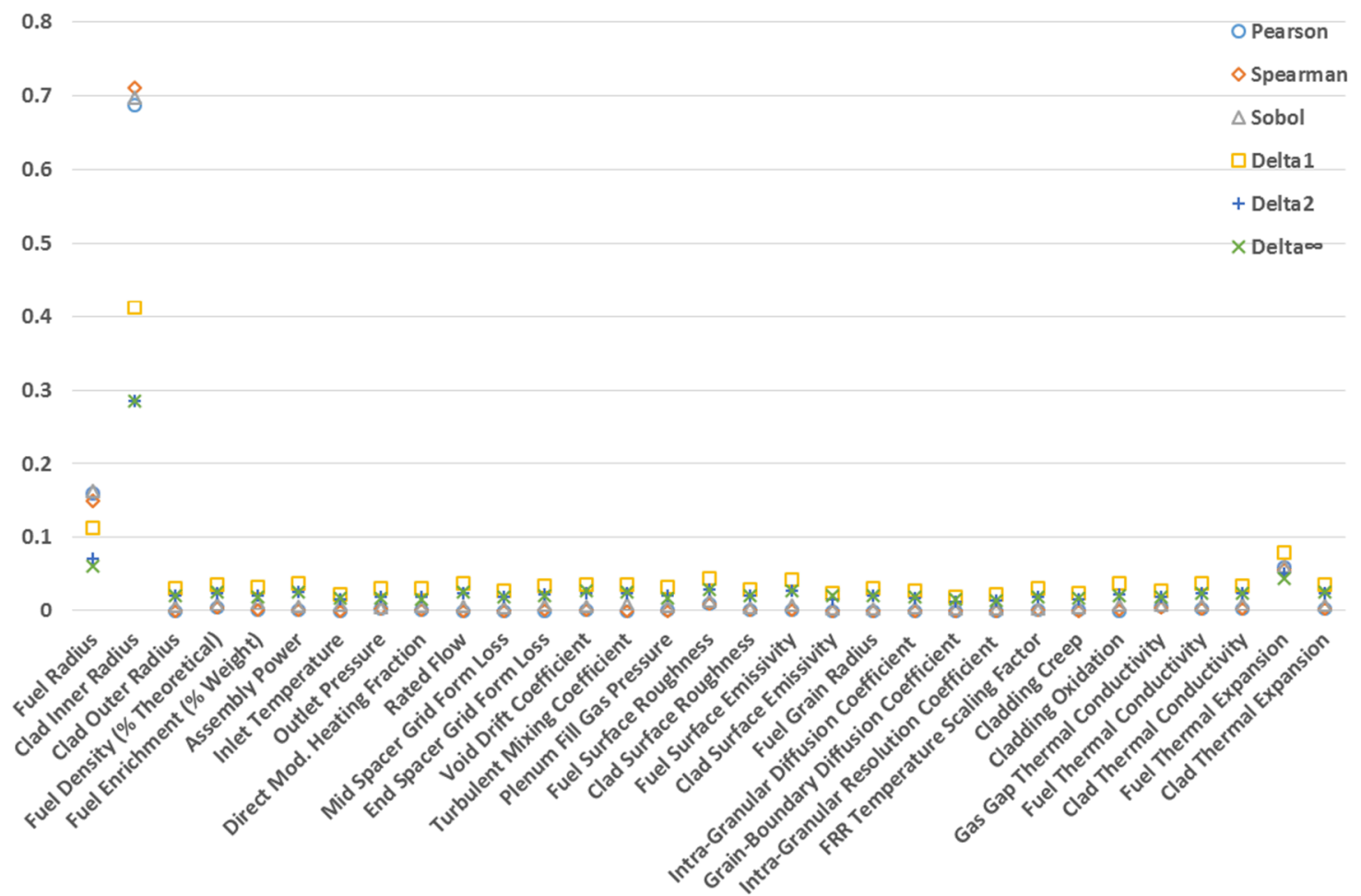

Figure 11. Sensitivity Analysis Measures for GCPP at 0 Days

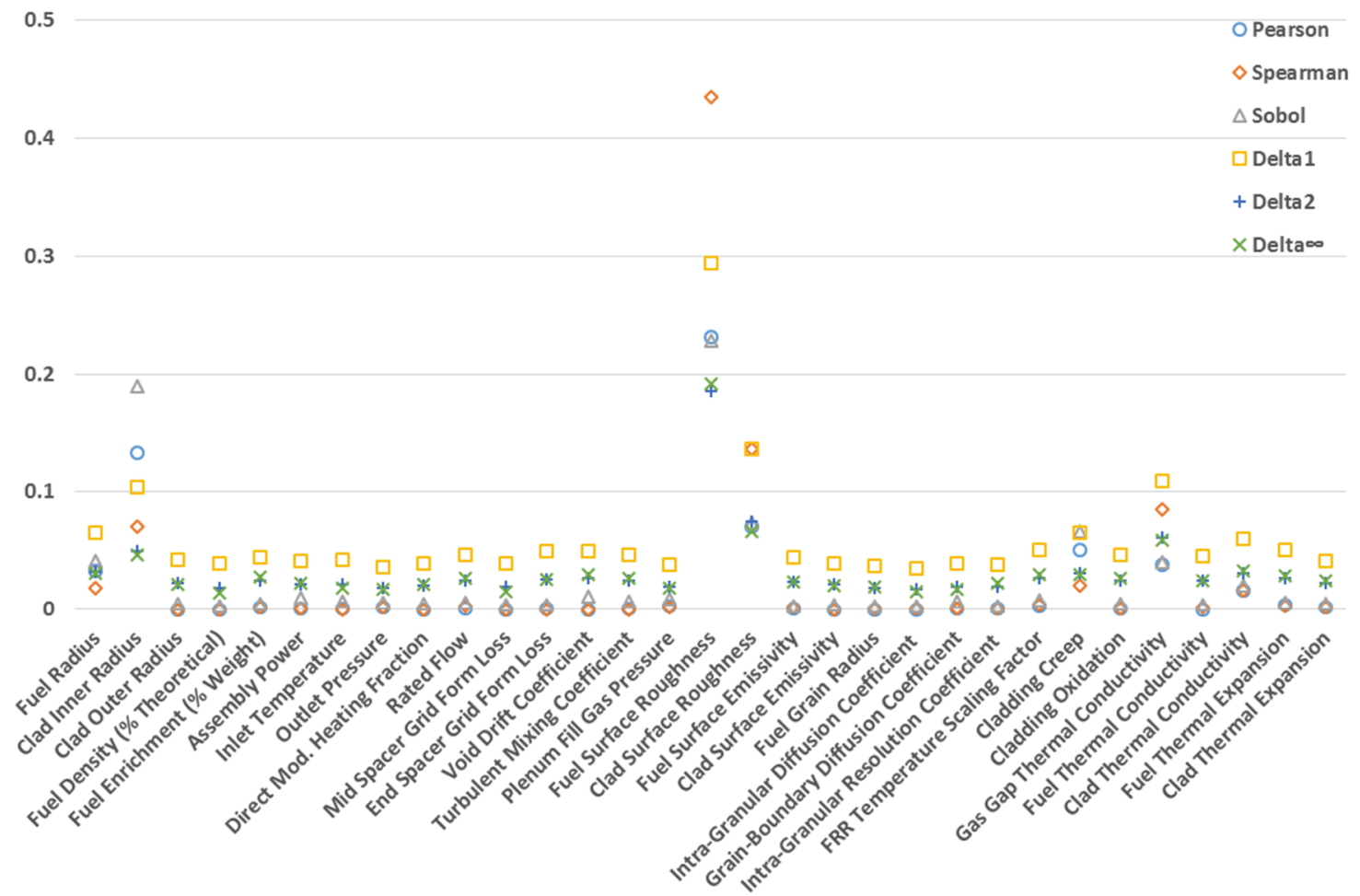

Figure 12. Sensitivity Analysis Measures for GCPP at 448 Days 
Figure 11 indicates that the clad inner radius and fuel radius are the largest contributors to GCPP uncertainty. This reaffirms that the GCPP is a strong function of gap dimensions. Note also that the third ranked input is the fuel thermal expansion due its direct effect on gap width. The Pearson squared summation is 1.01 indicating linear relations. The linearity of the data also creates consistent rankings among all measures.

Figure 12 contains a Pearson squared summation of 0.60 , indicating that while the majority of relations are still linear, $40 \%$ are nonlinear. These nonlinearities stem from the fact that while some cases still utilize the open gap models, the remainder have shifted to closed gap models. The presence of the open gap cases is evidenced by substantial SA measures for inner clad and fuel radii. Other cases have progressed to a complete independence from initial gap dimension, and a complete dependence on closed gap models. In such cases, GCPP is a strong function of fuel and clad roughness. This is due to BISON models treating closed gap cases as having finite thicknesses, which are highly dependent on fuel and cladding surface roughness.

Note that in Figure 12, Spearman strongly detects the fuel and cladding surface roughness in comparison to Pearson. This ability is attributable to the Spearman measures' superior ability to detect nonlinearities over Pearson. The fuel and surface roughness in particular are much larger with Spearman and Delta measures than Pearson and Sobol. This is likely due to the fact the perturbing of either roughness will only change GCPP for cases with small initial gap widths, and have next to no effect in instances with large initial gaps. This behavior is highly nonlinear, specifically non-additive.

The variance based Sobol indices closely follows Pearson, but in fact underperforms Spearman in detection of surface roughness relations. This indicates nonlinearities of the roughness stem not from individual terms alone (i.e. $Y=X^{2}$ ) in which Sobol indices are effective, but rather from interactions between inputs (i.e. $Y=X_{1} X_{2}$ ), which reaffirms that the model is non-additive.

It is worth noting that the GCPP uncertainty from the coupling of this work is more linear than the GCPP from past work with a VERA-CS and FRAPCON integration. This past work indicated that Pearson summations may be as low as 0.21 in mixed open and closed gap states [28]. Unfortunately a direct comparison between codes cannot be made at this time, as past FRAPCON work did not include the impactful fuel and cladding roughness as perturbed inputs. Nonetheless, the large increase in the summations of Pearson measures shows promise in establishing that within BISON, GCPP uncertainty behaves more linearly than its counterpart in FRAPCON due to more mechanistic models. This claim will be explored further in future work, by repeating a UQ/SA of VERA-CS/FRAPCON with the roughness inputs included.

Moving forward in the fuel lifecycle, Figures 13 and 14 show GCPP SA measures at 548 days and 1644 days respectively. 


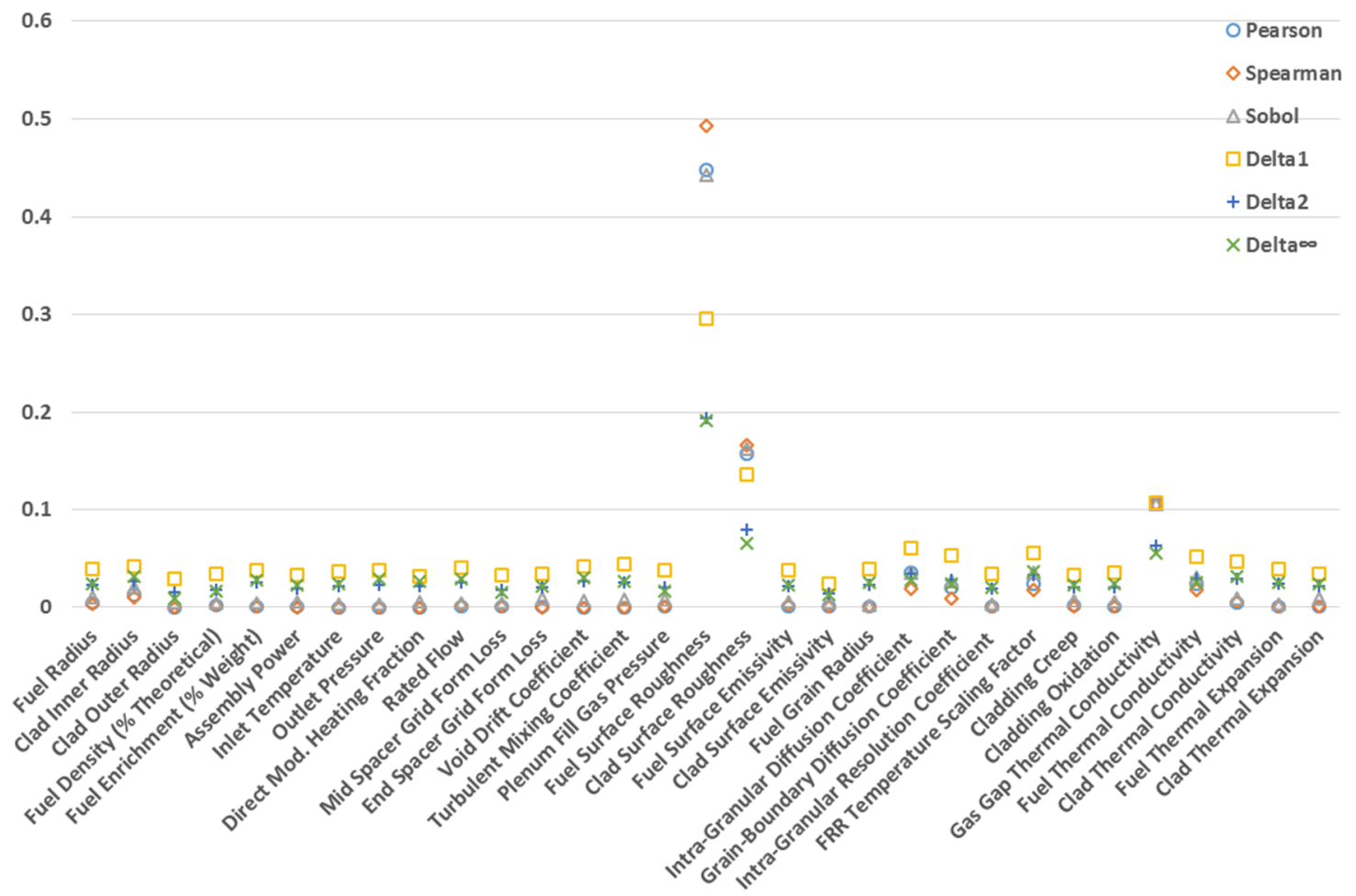

Figure 13. Sensitivity Analysis Measures for GCPP at 548 Days
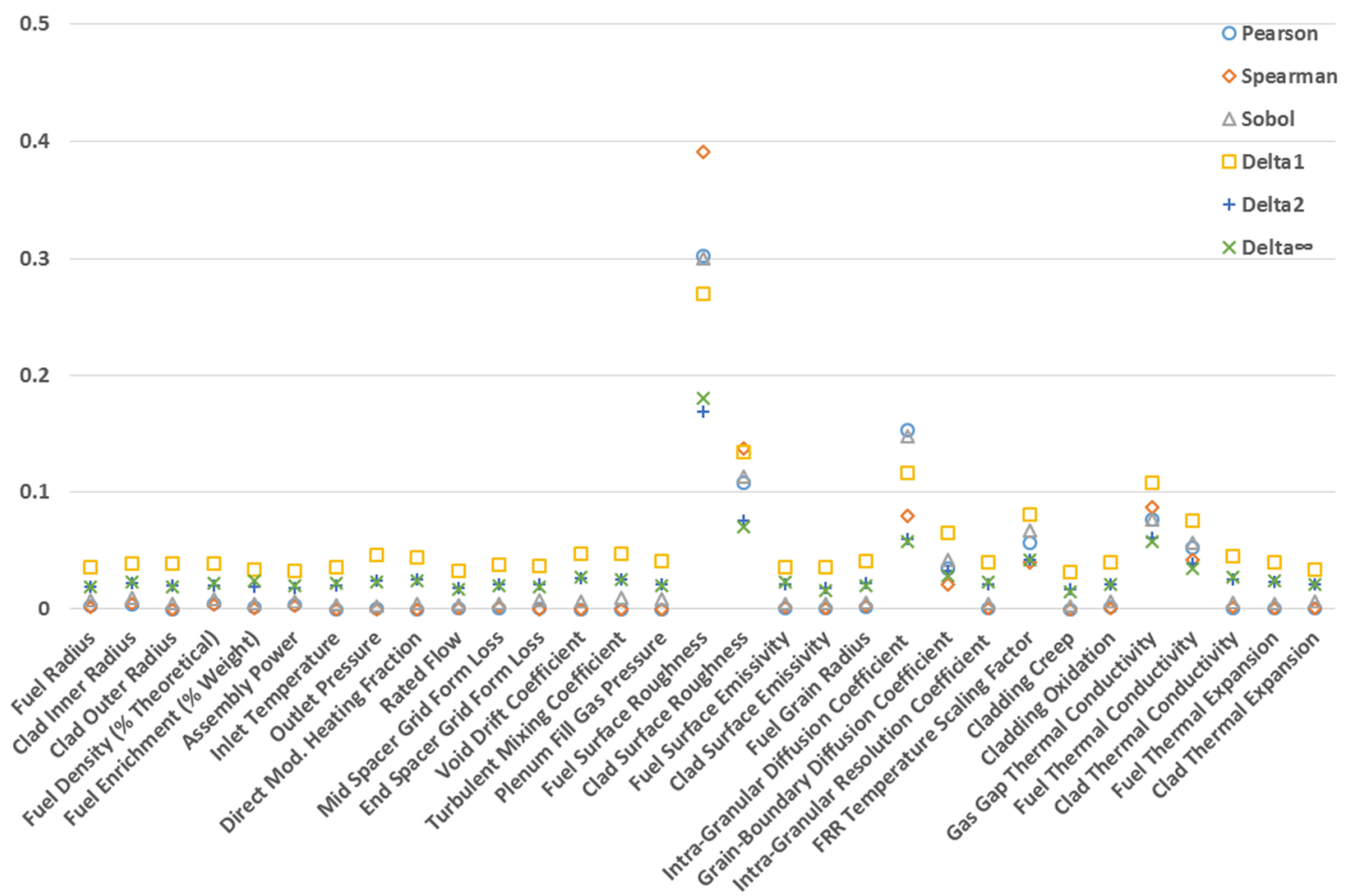

Figure 14. Sensitivity Analysis Measures for GCPP at 1643 Days 
The summation of Pearson measures for Figure 13 indicates that roughly $86 \%$ of uncertainties come from linear effects. The linearity is further evidenced by strong agreement between SA measures. Note a complete independence from fuel and cladding radii, indicating all cases are independent of initial gap dimensions at this state. The increase in SA measure for gap gas thermal conductivity is likely due to the small gap dimension used by BISON for closed gap model. Since gap gas conductance is calculated as the gas thermal conductivity over the gap width, smaller gap dimensions cause changes in gas gap thermal conductivity to be magnified.

The Pearson summation decreases to 0.81 for Figure 14 . This increase in nonlinearity can be explained by the increase in SA measures for all fission gas release inputs, namely the intra-granular and grain boundary diffusion coefficients as well as fission gas release temperature biasing. This indicates that at higher states, sufficient fission gas release has occurred to the point of altering gap gas thermal conductivity. The models associated with fission gas release are extremely complex, and the perturbation range of the diffusion coefficients is very large (see Table II). Thus the increase in nonlinearities from Figure 13 to Figure 14 is likely due to the increased role of fission gas products on GCPP at higher burnup.

\section{CONCLUSIONS}

LOTUS is a multiphysics environment which allows for the integration of the VERA-CS code with the fuel performance code BISON. All model inconsistencies are documented and either deemed negligible or given plans for future work. A successful Monte Carlo sampling with a direct data flow between the codes was used with a sample size of 2,500 VERA-CS cases and an equal number of BISON runs for the highest power pin at the final state.

Results provide UQ for MDNBR, MFCT, and GCPP for a 3 fuel cycle depletion case for a single assembly. Furthermore SA shows MDNBR to be linear, with little variation throughout the fuel cycle and a large dependence on assembly power and thermal hydraulic boundary conditions. MFCT also contains linear results, but shifts from a dependency on fuel thermal conductivity and gap dimensions, to almost exclusively fuel thermal conductivity after contact between fuel and cladding. Lastly, GCPP begins with linear relations and a strong dependence on gap dimension, followed by a nonlinear state dependent on gap dimension and surface roughness, followed by a predominantly linear state sensitive to surface roughness and fission gas release related inputs.

The SA results provide a path forward to decrease uncertainty for the FOM of this work. For instance, if a decrease in MFCT uncertainty at high burnup is desired, SA results of this work indicate that more accurate measurements and models of fuel thermal conductivity are necessary. While other sources of uncertainty exist, the results indicate that over $80 \%$ of uncertainty at higher burnup stems from fuel thermal conductivity alone. Thus if a decrease in MFCT uncertainty is desired, the primary focus must be on increasing the fidelity of fuel thermal conductivity models.

The UQ and SA results of MFCT and GCPP provide insights into the magnitude and behavior of the uncertainty associated with rod internal energy and gap conductance in general, both of which are significant inputs in LB-LOCA simulations. Future LB-LOCA based work aimed at reducing operation margins will use the aforementioned SA results as a means of selecting relevant inputs. The incorporation of rigorous UQ and SA methods into integrated high-fidelity simulations of VERA-CS and BISON can better inform irradiation experiments to substantially accelerate the qualification of new fuel design concepts such as accident tolerant fuel and higher burnup fuel. 


\section{ACKNOWLEDGMENTS}

We would like to thank the BISON team at INL for their extensive work in developing BISON. This work is supported by the U.S. Department of Energy, under DOE Idaho Operations Office Contract DE-AC0705ID14517. Accordingly, the U.S. Government retains a nonexclusive, royalty-free license to publish or reproduce the published form of this contribution, or allow others to do so, for U.S. Government purposes.

\section{REFERENCES}

[1] http://frapcon.labworks.org/, 2017.

[2] Sloan, B., McCorkle, D., and Bryden, K., "An Overview of Computational Environments for Engineering, Proc. 51st AIAA Aerospace Sciences Meeting including the New Horizons Forum and Aerospace Exposition, p. 285.

[3] https://www.casl.gov/, 2017.

[4] Gaston, D., Newman, C., Hansen, G., and Lebrun-Grandie, D., 2009, "MOOSE: A parallel computational framework for coupled systems of nonlinear equations," Nuclear Engineering and Design, 239(10), pp. 1768-1778.

[5] Todorova, N. K., and Ivanov, K. N., 2003, "Investigation of spatial coupling aspects for coupled code application in PWR safety analysis," Annals of Nuclear Energy, 30(2), pp. 189-209.

[6] Sjenitzer, B. L., Hoogenboom, J. E., Escalante, J. J., and Espinoza, V. S., 2015, "Coupling of dynamic Monte Carlo with thermal-hydraulic feedback," Annals of Nuclear Energy, 76, pp. 2739.

[7] Holt, L., Rohde, U., Seidl, M., Schubert, A., Van Uffelen, P., and Macián-Juan, R., 2015, "Development of a general coupling interface for the fuel performance code TRANSURANUSTested with the reactor dynamics code DYN3D," Annals of Nuclear Energy, 84, pp. 73-85.

[8] Hales, J., Tonks, M., Gleicher, F., Spencer, B., Novascone, S., Williamson, R., Pastore, G., and Perez, D., 2015, "Advanced multiphysics coupling for LWR fuel performance analysis," Annals of Nuclear Energy, 84, pp. 98-110.

[9] Porter, I., Knight, T. W., and Raynaud, P., 2015, "Potential Impacts of Modeling Full Reactor Cores Using Combined Fuel Performance and Thermal Hydraulics Codes," Nuclear Technology, 190(2), pp. 174-182.

[10] https://bison.inl.gov/ 2017.

[11] Folsom, C. P., Jensen, C. B., Williamson, R. L., Woolstenhulme, N. E., Ban, H., and Wachs, D. M., 2016, "BISON Modeling of Reactivity-Initiated Accident Experiments in a Static Environment," Idaho National Lab.(INL), Idaho Falls, ID (United States), INL/CON-16-37614.

[12] Magedanz, J., Avramova, M., Perin, Y., and Velkov, A., 2015, "High-fidelity multi-physics system TORT-TD/CTF/FRAPTRAN for light water reactor analysis," Annals of Nuclear Energy, 84, pp. 234-243.

[13] Chang, G. S., 2005, "MCWO-Linking MCNP and ORIGEN2 for Fuel Burnup Analysis," Idaho National Laboratory (INL), INEEL/CON-04-02085.

[14] Leppänen, J., Pusa, M., Viitanen, T., Valtavirta, V., and Kaltiaisenaho, T., 2015, "The Serpent Monte Carlo code: Status, development and applications in 2013," Annals of Nuclear Energy, 82, pp. 142-150. 
[15] Swiler, L. P., Lefebvre, R. A., Langley, B. R., and Thompson, A. B., 2017, "Integration of Dakota into the NEAMS Workbench," Sandia National Laboratories (SNL-NM), Albuquerque, NM (United States), SAND2017-7492.

[16] Bowman, S. M., 2011, "SCALE 6: Comprehensive Nuclear Safety Analysis Code System," Nuclear Technology, 174(2), pp. 126-148.

[17] Boyack, B., Catton, I., Duffey, R., Katsma, K., Lellouche, G., Levy, S., Wilson, G., and Zuber, N., 1990, "Quantifying reactor safety margins part 1: an overview of the code scaling, applicability, and uncertainty evaluation methodology," Nuclear Engineering and Design, 119(1), pp. 1-15.

[18] Marcum, W., and Brigantic, A., 2015, "Applying uncertainty and sensitivity on thermal hydraulic subchannel analysis for the multi-application small light water reactor," Nuclear Engineering and Design, 293, pp. 272-291.

[19] Ikonen, T., 2016, "Comparison of global sensitivity analysis methods-application to fuel behavior modeling," Nuclear Engineering and Design, 297, pp. 72-80.

[20] Ikonen, T., and Tulkki, V., 2014, "The importance of input interactions in the uncertainty and sensitivity analysis of nuclear fuel behavior," Nuclear Engineering and Design, 275, pp. 229-241. [21] Swiler, L. P., Gamble, K. A., Schmidt, R. C., and Williamson, R. L., 2015, "Sensitivity Analysis of OECD Benchmark Tests in BISON," Sandia National Laboratories (SNL-NM), Albuquerque, NM (United States), SAND2015-8808.

[22] Pastore, G., Swiler, L., Hales, J. D., Novascone, S. R., Perez, D. M., Spencer, B. W., Luzzi, L., Van Uffelen, P., and Williamson, R. L., 2015, "Uncertainty and sensitivity analysis of fission gas behavior in engineering-scale fuel modeling," Journal of Nuclear Materials, 456, pp. 398-408. [23] Talbot, P. W., Gamble, K. A., Rabiti, C., Wang, C., Alfonsi, A., Cogliati, J., Mandelli, D., and Prinja, A., 2016, "Time-Dependent Sensitivity Analysis of OECD Benchmark using BISON and RAVEN " Transactions of the American Nuclear Society.

[24] Brown, C., and Zhang, H., 2016, "Uncertainty quantification and sensitivity analysis with CASL Core Simulator VERA-CS," Annals of Nuclear Energy, 95, pp. 188-201.

[25] Brown, C., Zhang, H., Kucukboyaci, V., and Sung, Y., 2016, "Best estimate plus uncertainty analysis of departure from nucleate boiling limiting case with CASL core simulator VERA-CS in response to PWR main steam line break event," Nuclear Engineering and Design, 309, pp. 8-22. [26] Dinh, N., 2012, "CIPS validation data plan," Idaho National Laboratory (INL), INL/EXT-1225347.

[27] Williams, M. L., and Rearden, B. T., 2008, "SCALE-6 sensitivity/uncertainty methods and covariance data," Nuclear Data Sheets, 109(12), pp. 2796-2800.

[28] Blakely, C., Zhang, H., and Ban, H., 2018, "Sensitivity analysis of VERA-CS and FRAPCON coupling in a multiphysics environment," Annals of Nuclear Energy, 111, pp. 683-701.

[29] Borgonovo, E., 2007, "A new uncertainty importance measure," Reliability Engineering \& System Safety, 92(6), pp. 771-784.

[30] Zhai, Q., Yang, J., Xie, M., and Zhao, Y., 2014, "Generalized moment-independent importance measures based on Minkowski distance," European Journal of Operational Research, 239(2), pp. 449-455.

[31] Kochunas, B., Collins, B. S., Jabaay, D., Kim, K. S., Graham, A., Stimpson, S., Wieselquist, W. A., Clarno, K. T., Palmtag, S., and Downar, T., "VERA core simulator methodology for PWR cycle depletion, Oak Ridge National Laboratory (ORNL); Oak Ridge Leadership Computing Facility (OLCF); Consortium for Advanced Simulation of LWRs (CASL)," Proc. ANS MC2015, April 19-23, 2015, Nashville, TN. 
[32] Rempe, K., Smith, K., and Henry, A., 1989, "SIMULATE-3 pin power reconstruction: methodology and benchmarking," Nuclear Science and Engineering, 103(4), pp. 334-342.

[33] Novascone, S. R., Hales, J. D., Gardner, R., Pawlowski, R., Pastore, G., Toth, A., Clarno, K. T., Collins, B. S., Stimpson, S. G., and Powers, J. J., 2017, "Demonstration of Coupled Tiamat Single Assembly Calculations," Oak Ridge National Lab.(ORNL), Oak Ridge, TN (United States), CASL-U-2017-1357-000.

[34] Williamson, R., Hales, J., Novascone, S., Tonks, M., Gaston, D., Permann, C., Andrs, D., and Martineau, R., 2012, "Multidimensional multiphysics simulation of nuclear fuel behavior," Journal of Nuclear Materials, 423(1), pp. 149-163.

[35] Bai, X.-M., Tonks, M. R., Zhang, Y., and Hales, J. D., 2016, "Multiscale modeling of thermal conductivity of high burnup structures in UO 2 fuels," Journal of Nuclear Materials, 470, pp. 208215.

[36] Schwen, D., Aagesen, L. K., Peterson, J. W., and Tonks, M. R., 2017, "Rapid multiphasefield model development using a modular free energy based approach with automatic differentiation in MOOSE/MARMOT," Computational Materials Science, 132, pp. 36-45.

[37] Bratton, R. N., Jessee, M. A., and Wieselquist, W. A., 2015, "Rod internal pressure quantification and distribution analysis using FRAPCON," Oak Ridge National Laboratory (ORNL), Oak Ridge, TN (United States), ORNL/TM-2015/557.

[38] Stimpson, S., Powers, J., Gardner, R., Clarno, K., and Pawlowski, R., "Effect of Clad Fast Neutron Flux Distribution on Quarter-Core Fuel Performance Calculations with BISON, Proc. 2017 Water Reactor Fuel Performance Meeting, Sept. 10 - 14, Jeju Island, Korea.

[39] http://www.oecd-nea.org/tools/abstract/detail/ccc-0371, 2017.

[40] https://ec.europa.eu/jrc/en/scientific-tool/transuranus, 2017.

[41] http://www.nrc.gov/reading-rm/doc-collections/nuregs/contract/cr6150/v4/, 2017.

[42] Harding, B., Tremblay, C., and Cousineau, D., 2014, "Standard errors: A review and evaluation of standard error estimators using Monte Carlo simulations," Quant Methods Psychol, 10, pp. 107-123.

[43] Plischke, E., 2012, "An adaptive correlation ratio method using the cumulative sum of the reordered output," Reliability Engineering \& System Safety, 107, pp. 149-156.

[44] https://salib.readthedocs.io/en/latest/, 2017.

[45] Plischke, E., Borgonovo, E., and Smith, C. L., 2013, "Global sensitivity measures from given data," European Journal of Operational Research, 226(3), pp. 536-550.

[46] Botev, Z. I., Grotowski, J. F., and Kroese, D. P., 2010, "Kernel density estimation via diffusion," The Annals of Statistics, 38(5), pp. 2916-2957.

[47] http://pyqt-fit.readthedocs.io/en/latest/modules.html, 2017. 